Lindomar Soares dos Santos

Implementação de um sistema dosimétrico termoluminescente para utilização em dosimetria in vivo em teleterapia com feixes de fótons de energia alta 
Lindomar Soares dos Santos

\section{Implementação de um sistema dosimétrico termoluminescente para utilização em dosimetria in vivo em teleterapia com feixes de fótons de energia alta}

Dissertação apresentada à Faculdade de Filosofia, Ciências e Letras de Ribeirão Preto da Universidade de São Paulo para obtenção do título de Mestre em Ciências. Área de concentração: Física Aplicada à Medicina e Biologia

Orientador: Prof. Dr. Alessandro Martins da Costa 
AUTORIZO A REPRODUÇÃO E DIVULGAÇÃO TOTAL OU PARCIAL DESTE TRABALHO, POR QUALQUER MEIO CONVENCIONAL OU ELETRÔNICO, PARA FINS DE ESTUDO E PESQUISA, DESDE QUE CITADA A FONTE.

FICHA CATALOGRÁFICA

Santos, Lindomar Soares dos.

Implementação de um sistema dosimétrico termoluminescente para utilização em dosimetria in vivo em teleterapia com feixes de fótons de energia alta. Ribeirão Preto, 2007.

50 p.: il.; $30 \mathrm{~cm}$

Dissertação de Mestrado, apresentada à Faculdade de Filosofia, Ciências e Letras de Ribeirão Preto/USP. Área de concentração: Física Aplicada à Medicina e Biologia.

Orientador: Costa, Alessandro Martins da.

1. Radioterapia. 2. Garantia da qualidade. 3. Dosimetria termoluminescente. 4. Dosimetria in vivo. 5. Feixes de fótons. 


\section{FOLHA DE APROVAÇÃO}

Lindomar Soares dos Santos

Implementação de um sistema dosimétrico termoluminescente para utilização em dosimetria in vivo em teleterapia com feixes de fótons de energia alta

Dissertação apresentada à Faculdade de Filosofia, Ciências e Letras de Ribeirão Preto da Universidade de São Paulo para obtenção do título de Mestre em Ciências. Área de concentração: Física Aplicada à Medicina e Biologia.

Aprovado em:

Banca Examinadora

Prof. Dr.

Instituição:

Assinatura:

Prof. Dr.

Instituição:

Assinatura:

Prof. Dr.

Instituição: Assinatura: 


\section{DEDICATÓRIA}

Ao meus pais, Waldemar e Maria, ao meu irmão, Viumar, e aos grandes amigos, Edison e Simoni. 


\section{AGRADECIMENTOS}

Minha gratidão, que se estende a todos os que, de alguma forma, colaboraram para esta que foi a mais agradável fase de minha vida acadêmica:

Ao meu orientador, o Prof. Dr. Alessandro Martins da Costa, pela paciência, pela amizade e pelas inabaláveis serenidade e solicitude com que, desde o início e com muita compreensão, mesmo não me conhecendo bem, sempre me atendeu, ajudou e demonstrou confiança.

Aos professores do Departamento de Física e Matemática, pela agradável convivência, e, em especial, à Prof ${ }^{a}$ Dra. Patrícia Nicolucci, pela atenção, pela receptividade e pela objetividade com que me ajudou e sanou algumas de minhas dúvidas e temores.

Aos funcionários do Departamento do Física e Matemática, em especial aos Srs. Élcio Navas e José Aziani, pela convivência e apoio técnico.

Ao pessoal do Serviço de Radioterapia do Hospital das Clínicas da Faculdade de Medicina de Ribeirão Preto: Mizael, Fábio, Leonardo, Marlene, Edvaldo, Gustavo, Edenyse, Harley, Beatriz, Simone, Leandro, Vilma e Rita, pela boa convivência e ajuda.

Aos funcionários do CIDRA (Centro de Instrumentação, Dosimetria e Radioproteção), em especial à Márcia Carvalho, pela grande ajuda e invariável prontidão.

Ao alunos da disciplina "Eletromagnetismo e Óptica": Amanda, André, Daniela, Cristina, Drielly, Fernanda, Henrique, Jacqueline, Karmel, Lilian, Nathália e Wellington, por me proporcionarem uma nova e profícua experiência no Estágio Supervisionado em Docência.

Ao amigos do programa de pós-graduação: Mirko, Moisés, Alexandre, Marina, Ernando, Mayler, Lucas Baggio, Tiago, Lucas Oliveira, Alessandra, Diego, Rondinele, João (Borim), Jorge, Adelson, Fabiano, Luiz, Silvio, Lúcio e Ana Paula, pela agradável convivência.

Ao amigo Ailton Watanabe, por não ter medido esforços quando, atenciosa e cuidadosamente, me recebeu e me ciceroneou em minha vinda a Ribeirão Preto.

Aos amigos Patrícia Venturini e Alexandre Yoshida, pela confiança, pelo respeito e pelo apoio, que resistiram às adversidades e à distância.

Aos amigos Mônica Campiteli e Rodrigo Rotta, por todo apoio, pela sinceridade, pela confiança, pela paciência, pela acolhida e por insistirem em me fazer acreditar que eu não estava só.

Ao amigo Marco Antônio Nunes, pelo apoio, por nunca ter hesitado em me ajudar nos momentos difíceis e por ter, desde que o conheci, acreditado em mim, em meu discurso e em minhas intenções, apesar dos poucos motivos aparentes.

Ao Djalma, pela imprescindível colaboração e por ter estado presente e à disposição em todas as vezes que precisei.

À CAPES (Coordenação de Aperfeiçoamento de Pessoal de Nível Superior), pela concessão da bolsa de mestrado, e à FAPESP (Fundação de Amparo à Pesquisa do Estado de São Paulo), pelo apoio financeiro para realização desta pesquisa. 
À Universidade de São Paulo (USP), pela infra-estrutura oferecida.

Ao meus queridos pais, Waldemar e Maria, pelo empenho na formação de uma família íntegra, pelo apoio, pela imensa paciência e pela confiança cega e incondicional demonstrada em todas as fases da minha vida.

Ao meu irmão, Viumar, pela importante aproximação e por ter se feito prestativo, solícito e amigo.

Aos amigos, Edison e Simoni Penteado (inteligentes, exigentes e grandes exemplos de retidão), pela amizade comprovadamente sincera, pela lealdade incontestável, pela paciência sobre-humana, pela confiança, pela incansável ajuda em todos os momentos mais difíceis da minha vida, pelo incentivo, por terem se tornado minha maior referência de conduta ao resgatarem minhas crenças no que julgava ser correto e possível, por terem me ajudado a me tornar uma pessoa melhor e por não terem desistido de mim nem permitido que eu desistisse.

A todos os que colaboraram para a realização deste trabalho e para o meu aprimoramento pessoal e profissional. 
"A falta de liberdade não consiste jamais em estar segregado, e sim em estar em promiscuidade, pois o suplício inefável é não se poder estar sozinho".

Fyodor Mikhailovich Dostoevsky 


\section{Resumo}

A dosimetria in vivo é a verificação final da dose real administrada ao paciente e tornou-se atualmente necessária devido ao aumento da complexidade e da sofisticação das técnicas radioterápicas.

A finalidade deste trabalho foi apresentar, verificar e avaliar alguns procedimentos básicos, práticos e viáveis para a implementação da dosimetria in vivo com dosímetros termoluminescentes na verificação de dose em um serviço de radioterapia.

Para o estabelecimento do sistema dosimétrico termoluminescente, alguns testes e medições foram realizados, incluindo o procedimento de inicialização, a determinação da homogeneidade do grupo de dosímetros, a determinação do fator de correção individual de cada dosímetro, a determinação da faixa de linearidade do sistema e do coeficiente de calibração.

Medições em um objeto simulador antropomórfico foram realizadas para garantir que os métodos utilizados são satisfatórios antes que estes fossem usados para medições em pacientes. Medições de dose em um paciente foram feitas em um tratamento de câncer de próstata.

A metodologia proposta pode ser usada como parte de um programa de garantia de qualidade em um serviço de radioterapia. 


\section{Abstract}

In vivo dosimetry is the ultimate check of the actual dose delivered to an individual patient and has become a procedure actually necessary due to increasing complexity and sophistication of radiotherapy techniques.

The purpose of the present work was to present, verify and evaluate some basic, practical and viable procedures for the implementation of in vivo dosimetry with thermoluminescent dosimeters for patient dose verification at a radiotherapy service.

For the setting up of the thermoluminescent dosimetric system, several tests and measurements were carried out including the initialisation procedure, the determination of the batch homogeneity, the determination of individual correction factor of each dosimeter, the determination of linearity range of the system and its calibration coefficients.

Anthropomorphic phantom measurements were taken to ensure that the methods are satisfactory before they are used for patients measurements. Patient dose measurements were carried out in a prostate cancer treatment.

The proposed methodology can be used as a part of a quality assurance program in a radiotherapy service. 


\section{Sumário}

1 Introdução $\quad$ p. 12

1.1 Dosimetria TL . . . . . . . . . . . . . . . . . . p. 16

1.1 .1 Princípios básicos $\ldots \ldots \ldots \ldots \ldots \ldots \ldots$

1.1 .2 Dosímetros TL . . . . . . . . . . . . . . . . . . p. 19

2 Materiais e métodos $\quad$ p. 22

2.1 Estabelecimento do sistema dosimétrico TL . . . . . . . . . . . . p. 22

2.1.1 Procedimento de inicialização . . . . . . . . . . . . . p. 23

2.1.2 Homogeneidade do grupo . . . . . . . . . . . . . . . . . . p. 24

2.1.3 Dosímetros de referência e dosímetros de campo $\ldots \ldots \ldots$. . . p. 24

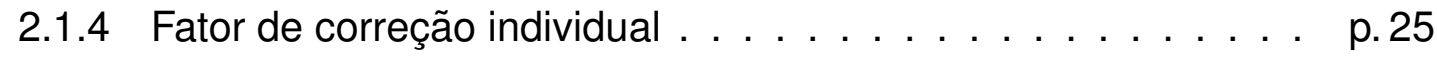

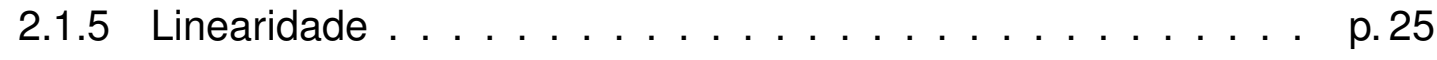

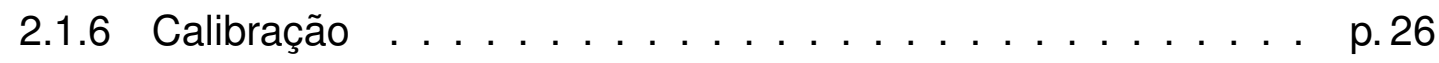

2.2 Medições no objeto simulador antropomórfico . . . . . . . . . . p.27

2.3 Medições in vivo $\ldots \ldots \ldots \ldots \ldots \ldots \ldots$

3 Resultados e Discussões $\quad$ p. 29

3.1 Estabelecimento do sistema dosimétrico TL . . . . . . . . . . . . . . p. 29

3.1.1 Procedimento de inicialização . . . . . . . . . . . . . . . . p. 29

3.1.2 Homogeneidade do grupo . . . . . . . . . . . . . . p.32

3.1.3 Fator de correção individual . . . . . . . . . . . . . . p.35

3.1 .4 Linearidade . . . . . . . . . . . . . . . . p.38 
3.2 Medições no objeto simulador antropomórfico . . . . . . . . . . . . . . p. 39

3.3 Medições in vivo . . . . . . . . . . . . . . . . . . . . . . p. 40

4 Conclusões

p. 43

Referências Bibliográficas

p. 44 


\section{Introdução}

A radioterapia é freqüentemente utilizada no tratamento do câncer, seja como uma modalidade simples ou em combinação com outras modalidades, como a cirurgia e a quimioterapia e utiliza radiações ionizantes baseando-se na destruição das células tumorais pela absorção da energia da radiação incidente. $O$ tratamento no qual a fonte de radiação é posicionada externamente ao paciente é a forma mais comum de radioterapia e é chamada de teleterapia, normalmente realizada com feixes de fótons (mais comumente raios $X$ de energias altas produzidos por um acelerador linear) e feixes de raios gama de unidades de cobalto e raios $X$ de energias menores na faixa de quilovoltagem. Além disso, feixes de elétrons de megavoltagem produzidos por um acelerador linear também são utilizados ${ }^{(1)}$. A utilização de aceleradores lineares na dupla modalidade (feixes de fótons e elétrons) pode substituir os equipamentos de raios $X$ de quilovoltagem na maioria das aplicações clínicas em radioterapia, entretanto, os equipamentos de raios $X$ de quilovoltagem continuam a ser utilizados, pois apresentam custo baixo quando comparados com unidades de megavoltagem, e porque o custo da terapia com elétrons pode ser proibitivo para alguns Serviços de Radioterapia ${ }^{(2)}$.

O principal problema da radioterapia é que as células tumorais não são tratadas isoladamente, isto é, o dano da radiação não é restrito somente às células tumorais, mas afeta também as células normais. Alguns dos avanços atuais na teleterapia surgiram com o uso crescente do computador como ferramenta de planejamento tridimensional do tratamento e controle da unidade de tratamento, que permite que a região de dose alta possa ser ajustada ao volume alvo em três dimensões. Os avanços das técnicas de imagem, tais como a tomografia computadorizada com raios $\mathrm{X}$ e a ressonância magnética, permitiram uma definição mais precisa dos volumes tumorais e das estruturas críticas, o que levou muitos centros a hoje desenvolverem e utilizarem técnicas de conformação do volume de dose alta e de escalonamento da dose, baseadas em blocos estáticos e colimadores multilâminas. Estas técnicas requerem métodos mais sofisticados de planejamento do tratamento e, com computadores cada 
vez mais rápidos, os métodos Monte Carlo estão agora na proximidade de se tornarem viáveis como base para o planejamento do tratamento de rotina, ao mesmo tempo em que técnicas de radioterapia de intensidade modulada em desenvolvimento requerem métodos mais sofisticados de verificação e garantia da qualidade ${ }^{(1)}$.

A exatidão e a precisão da dose no tumor e nos tecidos sadios adjacentes é um aspecto da radioterapia que deve ser considerado. Vários estudos indicam uma forte inclinação das curvas dose-resposta, tanto para as de controle do tumor como para as de complicações dos tecidos sadios adjacentes ${ }^{(3-7)}$, que servem de guia para especificação da exatidão da dose no tumor. Baseados na inclinação e na separação destas curvas, vários grupos têm proposto a necessidade de uma exatidão de -5$+7 \%$, com uma incerteza combinada, na distribuição da dose absorvida durante o tratamento ${ }^{(8-11)}$. Se as inclinações das curvas dose-resposta são pequenas, a exatidão pode ser menor, mas se as inclinações forem grandes, a exatidão requerida provavelmente é maior.

O plano de tratamento para um paciente é o resultado de um trabalho cuidadoso e da interação entre o físico médico e o radiooncologista. Em cada uma das muitas etapas no planejamento e na execução do tratamento são introduzidas incertezas que contribuem para a incerteza geral na dose absorvida distribuída aos pacientes ${ }^{(8,11-13)}$. É extremamente útil quantificar a incerteza geral na dose distribuída aos pacientes tratados de acordo com as técnicas freqüentemente aplicadas. Esta incerteza geral pode ser considerada como a exatidão máxima alcançável na distribuição de dose, contudo, para pacientes individuais, a exatidão na distribuição de dose pode ser menor que o desejável devido ao surgimento de erros sistemáticos na distribuição de dose, relacionados à influência dos contornos, movimento, inomogeneidades e variação da anatomia interna do paciente ${ }^{(14-17)}$. Além disso, incertezas podem ser introduzidas pela transferência dos dados do sistema de planejamento do tratamento ou do simulador para o acelerador por erros nos parâmetros da unidade de tratamento ou no posicionamento do paciente e dos modificadores de feixe ${ }^{(18,19)}$. A verificação final da dose real distribuída a um paciente individual somente pode ser realizada por meio da dosimetria in vivo, que é, talvez, a maneira mais óbvia de verificar a exatidão do tratamento do paciente e que, por isso, é recomendada por várias organizações nacionais e internacionais ${ }^{(8,20-23)}$. A publicação da Organização Mundial de Saúde sobre garantia da qualidade em radioterapia ${ }^{(21)}$ recomenda que a dosimetria in vivo deve ser realizada em todos os pacientes. 
A dosimetria in vivo pode ser feita colocando-se dosímetros sobre a pele ou em cavidades naturais do paciente. Se o dosímetro é posicionado sobre a pele, as doses de entrada e de saída podem ser medidas e, pela combinação das duas, a dose no interior do paciente pode ser obtida utilizando-se várias relações ${ }^{(14,24-29)}$. Em geral, as medições de dose de entrada servem para verificar a calibração da unidade de tratamento, a exatidão do posicionamento do paciente e dos modificadores de feixe e o cálculo do número de unidades monitoras. As medições de dose de saída servem para, além disso, verificar o alinhamento do feixe, verificar o processo de cálculo de dose relativa e para determinar a influência das variações de forma, tamanho e densidade do paciente no procedimento de cálculo de dose. Em situações em que a exatidão requerida na dose no tumor é alta, como nas técnicas de conformação do volume de dose alta e escalonamento da dose, os valores experimental e prescrito de dose no interior do paciente devem ser comparados.

Assim, um primeiro possível propósito da dosimetria in vivo é comparar as doses derivadas do sinal dos dosímetros posicionados sobre a pele com os valores teóricos calculados pelo sistema de planejamento de tratamento. Como, entretanto, um cálculo exato da dose na pele é difícil, e em muitos casos irrelevante, o sinal do dosímetro é convertido para dose em um ponto que ainda é próximo à pele, mas que está a uma certa profundidade, em que a exatidão do sistema de planejamento de tratamento é muito mais satisfatória. Com um ponto é próximo à superfície de entrada do feixe e outro próximo à superfície de saída, as doses correspondentes são a dose de entrada e dose de saída, respectivamente ${ }^{(24,25,30,31)}$.

Um propósito mais ambicioso da dosimetria in vivo é verificar a dose no volume alvo ${ }^{(32)}$ a fim de confirmar a exatidão da irradiação, o que é impossível, exceto quando os dosímetros podem ser introduzidos em cavidades naturais do corpo, tais como o esôfago, o reto, a vagina, etc. De fato, uma verificação da dose de entrada e da dose de saída é também uma verificação da dose no volume alvo ${ }^{(14,24-29)}$, contudo, se é observado um desvio entre o valor de dose de entrada ou dose de saída calculado e o valor medido, assumindo-se que o valor experimental está correto, pode-se supor que a dose no volume alvo está errada, que o cálculo das doses de entrada e saída está errado ou que haja uma combinação dos dois tipos de erro, o que torna de grande interesse uma verificação mais seletiva da dose no volume alvo.

Um terceiro possível propósito da dosimetria in vivo pode ser a determinação da dose na pele. Esta medição é crítica e requer uma metodologia especial ${ }^{(33-36)}$. 
A dosimetria TL (termoluminescente) e a dosimetria com diodos são as duas técnicas mais bem estabelecidas utilizadas para dosimetria in vivo ${ }^{(25,31,37-47)}$ e a escolha entre as duas pode depender de muitos fatores, como disponibilidade, características intrínsecas do tipo de detector, treinamento do pessoal, considerações financeiras e, claro, preferência pessoal ${ }^{(42)}$.

As câmaras de ionização apresentam algumas desvantagens que as tornam inapropriadas para a dosimetria in vivo: são volumosas e frágeis e, portanto, não são facilmente usadas em pacientes, além de requerem uma conexão a um eletrômetro e a aplicação de uma alta voltagem, que pode ser um risco de choque elétrico para o paciente. Conseqüentemente, as câmaras de ionização são atualmente recomendadas para um número muito limitado de aplicações tal como irradiação de corpo inteiro ${ }^{(34)}$.

Algumas outras técnicas também têm sido testadas para dosimetria in vivo, mas ainda não estão em uso clínico rotineiro, como por exemplo a dosimetria com alanina ${ }^{(48)}$, a dosimetria com transistores de efeito de campo metal-óxido-semicondutor (MOSFET) ${ }^{(49-51)}$, a dosimetria com cintiladores plásticos ${ }^{(52-54)}$, a dosimetria com diamantes ${ }^{(55-57)}$, a técnica da luminescência opticamente estimulada ${ }^{(58-61)}$, a dosimetria com géis ${ }^{(47,62)}$ e a dosimetria com filmes portais ou portais eletrônicos ${ }^{(43,45,47,63)}$.

O objetivo geral desta proposta é desenvolver e implantar um protocolo de dosimetria in vivo em teleterapia com feixes de fótons, utilizando a técnica da dosimetria $\mathrm{TL}$, tendo como meta bem definida melhorar a qualidade dos tratamentos.

Os objetivos específicos foram o estabelecimento do sistema dosimétrico TL, a utilização da dosimetria TL na verificação da dose prescrita aos pacientes em um Serviço de Radioterapia e, também, o estabelecimento da dosimetria in vivo como parte de um programa de garantia da qualidade em um Serviço de Radioterapia.

Este trabalho foi desenvolvido no Serviço de Radioterapia do HCFMRP (Hospital das Clínicas da Faculdade de Medicina de Ribeirão Preto), USP (Universidade de São Paulo).

Uma das justificativas para a escolha da técnica da dosimetria TL foi a disponibilidade de um laboratório de instrumentação e dosimetria TL no CIDRA (Centro de Instrumentação, Dosimetria e Radioproteção) da FFCLRP (Faculdade de Filosofia, Ciências e Letras de Ribeirão Preto), USP. As características intrínsecas dos detectores TL permitiram que as irradiações fossem realizadas no HCFMRP e as avaliações dos dosímetros no CIDRA. A carga de trabalho exigida em um programa de dosime- 
tria in vivo depende de muitos fatores, tais como a exatidão requerida, a freqüência de verificações, o tempo dedicado à análise dos resultados e o pessoal envolvido. A avaliação dos detectores TL é realizada em um estágio posterior ao tratamento propriamente dito. É importante perceber que o método da dosimetria TL modifica muito pouco o procedimento clínico como um todo e somente apresenta uma maior carga de trabalho no processo de leitura dos detectores. Finalmente, a possibilidade de reutilização dos dosímetros TL é um importante aspecto de questão financeira.

\subsection{Dosimetria TL}

\subsubsection{Princípios básicos}

A dosimetria TL é fundamentada no princípio de que alguns cristais podem absorver e armazenar a energia da radiação ionizante, que é reemitida na forma de radiação eletromagnética, principalmente na região de comprimento de onda visível, quando estes cristais são aquecidos. A luz emitida é então detectada por uma fotomultiplicadora e correlacionada à dose absorvida recebida pelo material $\mathrm{TL}^{(64-68)}$.

Os materiais TL consistem de cristais que são em geral isolantes - em um modelo de bandas para os níveis de energia dos elétrons nos cristais, as bandas de energia mais baixas têm seus níveis totalmente preenchidos pelos elétrons. O nível mais alto preenchido (chamado de banda de valência) está separado do nível mais baixo da camada seguinte (chamado de banda de condução) por um intervalo proibido de níveis de energia (chamado de banda proibida), que possui uma largura de alguns eV para materiais TL típicos. À temperatura ambiente, a probabilidade de que um elétron consiga transpor a banda proibida é praticamente desprezível. Se um material TL é exposto à radiação ionizante, são produzidos pares de elétrons e buracos; os elétrons livres se deslocam através do cristal na banda de condução por um tempo curto, até serem capturados em estados metaestáveis de energia, localizados na banda proibida (chamados de armadilhas), se recombinarem radiativamente (fluorescência) ou não com os buracos na banda de valência ou serem capturados em centros de luminescência (provavelmente armadilhas muito profundas em relação à banda de condução) já ativados por buracos devido à irradiação, com a emissão de luz (radioluminescência). Os buracos criados pela radiação ionizante na estrutura do cristal comportam-se de maneira semelhante aos elétrons. 
Os elétrons podem ficar nas armadilhas por períodos prolongados (até meses), o que confere ao método TL a vantagem de armazenagem de informação, que pode ser coletada aquecendo-se o cristal a uma temperatura que depende da sua natureza. A energia sob a forma de calor é absorvida pelos elétrons, que escapam das armadilhas indo novamente para a banda de condução, que podem ser rearmadilhados, ou se recombinarem radiativamente ou não com os buracos na banda de valência ou se recombinarem radiativamente em centros de luminescência ativados por buracos, o que causa a emissão de luz, ou a termoluminescência. Os mesmos processos descritos para os elétrons podem ser analogamente discutidos para os buracos.

O aquecimento e a detecção de luz são realizados em um sistema de leitura chamado de leitora TL. A quantidade de luz emitida pelo material TL depende da temperatura e do número de elétrons armadilhados, que depende da dose absorvida no cristal, então a quantidade de luz emitida também depende da dose absorvida no cristal. $\mathrm{Na}$ maioria dos materiais TL há mais que um tipo de armadilha, com diferentes profundidades em relação à banda de condução e que, portanto, serão desocupadas em diferentes temperaturas. $O$ grande número de armadilhas leva a uma função complicada da intensidade da luz em função da temperatura, chamada de curva de emissão $T L$, que consiste de diferentes picos TL correspondentes a diferentes estados de energia no cristal. Estes picos podem ser instáveis, decaindo mais ou menos rapidamente com o tempo de acordo com o material TL considerado, ou estáveis. Um dosímetro TL sempre contém picos instáveis e estáveis, sendo os picos estáveis utilizados em dosimetria e chamados de picos dosimétricos.

Após a leitura, o material TL está completamente em seu estado original e, neste caso, pronto para ser reutilizado, ou precisa de um tratamento térmico especial para restaurar seu estado original ${ }^{(69)}$.

A fim de descrever as curvas de emissão TL matematicamente, tem-se que considerar que todas as armadilhas serão preenchidas com diferentes números de elétrons.

A probabilidade p por unidade de tempo de um elétron deixar uma armadilha, indo para a banda de condução, é dada por

$$
p=s \exp \left(-\frac{E}{k T}\right),
$$

em que s é o fator de freqüência $\left(\mathrm{s}^{-1}\right)$, que é uma constante específica da armadilha relacionada com as vibrações da rede cristalina, Ee é a profundidade da armadilha (eV), $k$ é a constante de Boltzmann $\left(\mathrm{eV} \cdot \mathrm{K}^{-1}\right)$ e T é a temperatura absoluta $(\mathrm{K})$. 
A suposição básica de que nenhum elétron que deixa a armadilha é rearmadilhado dá o conceito de cinética de primeira ordem. Se n é o número de elétrons armadilhados no material e se a temperatura $T$ é mantida constante, então $n$ decresce com o tempo $\mathrm{t}$ de acordo com a seguinte expressão:

$$
\frac{\mathrm{dn}}{\mathrm{dt}}=-\mathrm{pn}
$$

Usando a equação 1.1 e integrando a equação 1.2,

$$
\int_{n_{0}}^{n} \frac{d n}{n}=-\int_{0}^{t} s \exp \left(-\frac{E}{k T}\right) d t
$$

obtém-se

$$
\mathrm{n}=\mathrm{n}_{0} \exp \left[-\operatorname{st} \exp \left(-\frac{\mathrm{E}}{\mathrm{kT}}\right)\right],
$$

em que $\mathrm{n}_{0}$ é o número de elétrons armadilhados no instante $\mathrm{t}=0$.

Em 1945, Randall e Wilkins ${ }^{(70,71)}$ utilizaram extensivamente uma representação matemática para cada pico TL em uma curva de emissão supondo como uma primeira aproximação que o cristal é exposto a uma taxa de aquecimento $\beta=\mathrm{dT} / \mathrm{dt}$ constante e adotando uma cinética de primeira ordem. Assim, a intensidade da luz TL, I, a qualquer temperatura, é diretamente proporcional à taxa de desarmadilhamento:

$$
\mathrm{I}=-\mathrm{c} \frac{\mathrm{dn}}{\mathrm{dt}}=\mathrm{cpn},
$$

em que c é uma constante que pode ser fixada como uma unidade.

Usando a equação 1.4 obtemos:

$$
\mathrm{I}(\mathrm{t})=\mathrm{n}_{0} \mathrm{~s} \exp \left(-\frac{\mathrm{E}}{\mathrm{kT}}\right) \exp \left[-\mathrm{st} \exp \left(-\frac{\mathrm{E}}{\mathrm{kT}}\right)\right] .
$$

Utilizando $d t=d T / \beta$, a partir da equação 1.3 tem-se

$$
\int_{n_{0}}^{n} \frac{d n}{n}=-\frac{s}{\beta} \int_{T_{0}}^{T} \exp \left(-\frac{E}{k T^{\prime}}\right) d T^{\prime}
$$

e de novo

$$
\mathrm{n}=\mathrm{n}_{0} \exp \left[-\frac{\mathrm{s}}{\beta} \int_{\mathrm{T}_{0}}^{\mathrm{T}} \exp \left(-\frac{\mathrm{E}}{\mathrm{k} \mathrm{T}^{\prime}}\right) \mathrm{dT^{ \prime }}\right] .
$$

Usando a equação 1.5

$$
\mathrm{I}(\mathrm{T})=\mathrm{n}_{0} \operatorname{sexp}\left(-\frac{\mathrm{E}}{\mathrm{kT}}\right) \exp \left[-\frac{\mathrm{s}}{\beta} \int_{\mathrm{T}_{0}}^{\mathrm{T}} \exp \left(-\frac{\mathrm{E}}{\mathrm{kT} \mathrm{T}^{\prime}}\right) \mathrm{dT^{ \prime }}\right] .
$$


Esta expressão pode ser avaliada por integração numérica e produz uma curva em forma de sino com um máximo de intensidade a uma temperatura característica $\mathrm{T}_{\mathrm{M}}$.

Uma importante relação é obtida fixando

$$
\left(\frac{\mathrm{dl}}{\mathrm{dT}}\right)_{\mathrm{T}=\mathrm{T}_{\mathrm{M}}}=0,
$$

que produz a expressão

$$
\frac{\beta E}{k T_{M}^{2}}=\operatorname{sexp}\left(-\frac{E}{k T_{M}}\right) .
$$

A partir da equação 1.8, pode-se observar que: para uma taxa de aquecimento constante, $\mathrm{T}_{\mathrm{M}}$ aumenta com o aumento de $\mathrm{E}$ ou com a diminuição de s; para uma dada armadilha (E e s constantes), $\mathrm{T}_{\mathrm{M}}$ aumenta com o aumento da taxa de aquecimento; $T_{M}$ é independente de $n_{0}$.

A função dada pela equação 1.7 é muito complicada para ser utilizada diretamente para ajuste e análise da curva de emissão TL, no entanto, uma expressão simplificada pode ser utilizada para descrever a intensidade da luz TL em função da temperatura $^{(72)}$ :

$$
\mathrm{I}(\mathrm{T})=\mathrm{I}_{\mathrm{M}} \exp \left\{1+\mathrm{W}\left(\mathrm{T}-\mathrm{T}_{\mathrm{M}}\right)-\exp \left[\mathrm{W}\left(\mathrm{T}-\mathrm{T}_{\mathrm{M}}\right)\right]\right\},
$$

em que $I_{M}$ é o máximo de intensidade da luz $T L$ na temperatura $T_{M}$ e $W=E / k T_{M}^{2}$. Neste caso, a informação sobre os parâmetros de armadilhamento, s e no, é perdida, o que é de nenhuma importância na maior parte das aplicações dosimétricas. A equação 1.9 ou abordagens semelhantes ${ }^{(73,74)}$ podem ser usadas para análise matemática da curva de emissão.

\subsubsection{Dosímetros TL}

Os materiais TL geralmente utilizados para dosimetria in vivo são o fluoreto de lítio (LiF), o borato de lítio $\left(\mathrm{Li}_{2} \mathrm{~B}_{4} \mathrm{O}_{7}\right)$, o sulfato de cálcio $\left(\mathrm{CaSO}_{4}\right)$ e o fluoreto de cálcio $\left(\mathrm{CaF}_{2}\right)$, dopados com impurezas para a criação de armadilhas e centros de luminescência, como por exemplo o fluoreto de lítio dopado com magnésio e titânio ( $\mathrm{LiF}: \mathrm{Mg}, \mathrm{Ti}$ ), o fluoreto de lítio dopado com magnésio, cobre e fósforo ( $\mathrm{LiF}: \mathrm{Mg}, \mathrm{Cu}, \mathrm{P}$ ), o borato de lítio dopado com manganês $\left(\mathrm{Li}_{2} \mathrm{~B}_{4} \mathrm{O}_{7}: \mathrm{Mn}\right)$, o sulfato de cálcio dopado com disprósio $\left(\mathrm{CaSO}_{4}\right.$ : Dy), o fluoreto de cálcio dopado com disprósio $\left(\mathrm{CaF}_{2}\right.$ : Dy), etc. ${ }^{(42,44)}$ - todos disponíveis na forma de pó ou de dosímetros sólidos. A pequena dependência da 
resposta com a taxa de dose, temperatura e energia da radiação incidente na faixa terapêutica e a ampla faixa de dose em que podem ser aplicados, fazem com que os dosímetros TL sejam apropriados para os propósitos da dosimetria in vivo ${ }^{(63)}$.

Os dosímetros TL podem ser utilizados para medições de dose de entrada ou saída quando uma espessura de equilíbrio eletrônico é empregada. Os dosímetros TL também são úteis para medições de dose na superfície, já que, usando dosímetros TL de espessuras diferentes, a dose pode ser extrapolada para a superfície e interpolada entre as diferentes profundidades ${ }^{(75)}$ ou, com dosímetros TL com grafite, a dose na profundidade de, aproximadamente, $0,1 \mathrm{~mm}$ pode ser medida ${ }^{(76)}$.

As principais vantagens dos dosímetros TL são o tamanho pequeno e, portanto, sua boa resolução espacial, a sensibilidade alta e a ausência de alta voltagem e cabos. Alguns materiais TL podem ser considerados equivalentes ao tecido e outros equivalentes ao osso; a equivalência ao tecido é importante quando as medições são feitas sob condições de irradiação em que é difícil avaliar a energia efetiva dos fótons, como por exemplo na determinação de dose em órgãos críticos ${ }^{(35,75-79)}$. Contudo, deve-se observar que apesar da equivalência ao tecido de alguns materiais TL, eles normalmente têm uma densidade eletrônica diferente da densidade eletrônica da água e isto pode levar a uma pertubação do campo de radiação e alterar a dose registrada pelo detector ${ }^{(80)}$.

As principais desvantagens dos dosímetros TL são a impossibilidade de seu uso para dosimetria in vivo em tempo real, seu difícil manuseio e a dificuldade na identificação de um dosímetro individual ${ }^{(63)}$.

$\mathrm{O}$ material TL mais comumente usado em radioterapia é o $\mathrm{LiF}: \mathrm{Mg}, \mathrm{Ti}^{(45)}$, que tem uma equivalência ao tecido bastante apropriada (o número atômico efetivo do LiF : Mg, Ti é 8,14 e o do tecido é 7,4) e pode ser produzido nas formas desejadas, tais como pó, bastões ou discos com dimensões pequenas (da ordem de alguns milímetros). $\mathrm{O} \mathrm{CaSO}_{4}$ : Dy e o $\mathrm{CaF}_{2}$ : Dy são mais sensíveis que o $\mathrm{LiF}: \mathrm{Mg}, \mathrm{Ti}$, podem ser utilizados para medição de doses muito baixas e podem ser considerados equivalentes ao osso (o número atômico efetivo do $\mathrm{CaSO}_{4}$ : Dy é 15,6 e o do $\mathrm{CaF}_{2}$ : Dy é 16,6). $\mathrm{O} \mathrm{Li}_{2} \mathrm{~B}_{4} \mathrm{O}_{7}$ : Mn tem um número atômico efetivo igual ao do tecido, de modo que a sua sensibilidade não muda para fótons com energias maiores que $100 \mathrm{keV}$, e para energias menores, sua resposta diminui aproximadamente de $10-15 \%{ }^{(67)}$; as principais desvantagens do $\mathrm{Li}_{2} \mathrm{~B}_{4} \mathrm{O}_{7}$ : Mn são o fato dele ser higroscópico e muito sensível à radiação ultravioleta, e conseqüentemente difícil de usar ${ }^{(45)}$. O LiF : Mg, Cu,P é até 
30 vezes mais sensível que o $\mathrm{LiF}: \mathrm{Mg}, \mathrm{Ti}^{(45)}$ e apresenta a desvantagem de ser muito sensível a tratamentos térmicos incorretos ${ }^{(81)}$. Dessa forma, o LiF : Mg, Ti oferece maiores vantagens no uso rotineiro em radioterapia. 


\section{Materiais e métodos}

\subsection{Estabelecimento do sistema dosimétrico TL}

Um sistema dosimétrico TL consiste de várias partes: os elementos passivos (os dosímetros TL ou detectores); uma leitora TL, consistindo esquematicamente de um sistema de aquecimento, um tubo fotomultiplicador e os circuitos eletrônicos associados; um algoritmo apropriado para converter o sinal TL (resposta da leitora) para dose; fornos e/ou estufas para serem utilizados nos tratamentos térmicos dos dosímetros.

Foi utilizado um grupo com setenta e dois dosímetros TL de LiF : Mg, Ti com dimensões de $3,1 \times 3,1 \times 0,89 \mathrm{~mm}^{3}$, numerados com traços bem finos de grafite e sempre manipulados com uma pinça.

As avaliações dos dosímetros foram realizadas utilizando-se uma leitora TL manual da Harshaw, modelo $2000 \mathrm{~B}+\mathrm{C}$, com taxa de aquecimento constante de $8^{\circ} \mathrm{C} / \mathrm{s}$, pertencente ao CIDRA. A leitora possuía uma entrada de gás na câmara de avaliação TL, o que permitiu o uso de um fluxo constante de nitrogênio durante as avaliações com a intenção de reduzir a TL espúria e para aumentar a vida útil da prancheta do equipamento, aquecida uma corrente elétrica, sobre a qual é colocado o dosímetro TL. Cada ciclo de avaliação durou $25 \mathrm{~s}$ e a temperatura variou entre $50^{\circ} \mathrm{C}$ e $250^{\circ} \mathrm{C}$. A estabilidade da leitora foi testada utilizando-se a luz de referência do instrumento.

Os tratamentos térmicos dos dosímetros de $\mathrm{LiF}: \mathrm{Mg}$, Ti foram realizados nos fornos Bravac e estufas Olidef CZ também pertencentes ao CIDRA.

Todas as irradiações foram realizadas utilizando um acelerador linear clínico da Siemens, modelo Mevatron XII, com potencial de aceleração de $10 \mathrm{MV}$, pertencente ao Serviço de Radioterapia do HCFMRP, USP. Os dosímetros TL foram sempre irradiados com esta fonte sob $20 \mathrm{~mm}$ de acrílico ou $3 \mathrm{~mm}$ de latão, para a obtenção de equilíbrio eletrônico. 
Para o estabelecimento do sistema dosimétrico TL, foi necessário preencher alguns requisistos para inicialização, caracterização e calibração do material TL. Estas operações consistiram de vários testes e medições: procedimento de inicialização, determinação da homogeneidade do lote, determinação do fator de correção individual de cada dosímetro, determinação da faixa de linearidade do sistema e determinação dos coeficientes de calibração para a qualidade do feixe ${ }^{(42,43,45,68)}$.

Para a determinação dos coeficientes de calibração, foi utilizada uma câmara de ionização cilíndrica (tipo Farmer), à prova d'água, fabricada pela PTW-Freiburg, modelo TN30013, com volume sensível de $0,6 \mathrm{~cm}^{3}$, calibrada em termos de dose absorvida na água, acoplada a um eletrômetro também fabricado pela PTW-Freiburg, modelo UNIDOS E T10010. Também foi utilizado um objeto simulador geométrico de água com dimensões de $35 \times 35 \times 35 \mathrm{~cm}^{3}$ e um objeto simulador geométrico de acrílico com dimensões de $30 \times 30 \times 12,9 \mathrm{~cm}^{3}$, ambos pertencentes ao Serviço de Radioterapia do HCFMRP, USP.

A avaliação e a expressão das incertezas de medição foram feitas de acordo com as recomendações dadas no texto "Guia para a Expressão da Incerteza de Medição" (82).

\subsubsection{Procedimento de inicialização}

O procedimento de inicialização é recomendado para reduzir a possibilidade de variações nas características de desempenho dos dosímetros TL durante o uso ${ }^{(68)}$.

A primeira etapa do procedimento envolveu o tratamento térmico dos dosímetros utilizando os parâmetros (temperatura e tempo) indicados para o LiF : Mg, Ti. No final do procedimento de tratamento térmico os dosímetros foram avaliados para verificação do sinal de fundo na leitora TL, que teve sua estabilidade verificada antes e depois de todas as sessões de avaliação.

$\mathrm{O}$ tratamento térmico do $\mathrm{LiF}: \mathrm{Mg}$, Ti consistiu de um aquecimento de $1 \mathrm{~h}$ a $400^{\circ} \mathrm{C}$ seguido de outro aquecimento de $2 \mathrm{~h}$ a $100^{\circ} \mathrm{C}$, como recomendado pelo fabricante dos dosímetros. Neste caso, em que o tratamento térmico envolveu dois aquecimentos, os dosímetros tiveram de ser resfriados até a temperatura ambiente no final do primeiro aquecimento e depois colocados no forno pré-aquecido para o segundo aquecimento. $\mathrm{O}$ aquecimento de $1 \mathrm{~h} \mathrm{a} 400^{\circ} \mathrm{C}$ tem duas funções: minimizar o sinal TL residual esvaziando as armadilhas mais profundas que as do pico dosimétrico e restaurar o estado 
original da sensibilidade e da estrutura da curva de emissão. O propósito do aquecimento de $2 \mathrm{~h}$ a $100^{\circ} \mathrm{C}$ é reduzir o decaimento térmico do sinal TL minimizando a contribuição dos picos de baixa temperatura ${ }^{(67)}$.

\subsubsection{Homogeneidade do grupo}

Todos os setenta e dois dosímetros do grupo foram tratados termicamente, irradiados com uma mesma dose de $0,5 \mathrm{~Gy}$ e avaliados quatro vezes. Sendo R a emissão TL de cada dosímetro em cada série de avaliação, o índice de homogeneidade do grupo foi representado por ${ }^{(68)}$

$$
\Delta_{\max }=\frac{R_{\max }-R_{\min }}{R_{\min }} \cdot 100 \leq 30
$$

em que $R_{\max }$ e $R_{\min }$ são os valores máximos e mínimos de $R$ em cada série de avaliações. Caso algum dos $\Delta_{\max }$ obtidos fosse maior que 30 , alguns dosímetros TL teriam de ser rejeitados.

\subsubsection{Dosímetros de referência e dosímetros de campo}

A principal diferença entre os chamados dosímetros de referência e os dosímetros de campo é o seu uso.

Uma das funções dos dosímetros de referência é fornecer um resposta média, em relação à qual as respostas dos dosímetros de campo são normalizadas a fim de produzir os fatores de correção individuais (discutidos posteriormente), e a outra é determinar um único coeficiente de calibração para todos os dosímetros do grupo (também discutido posteriormente).

Os dosímetros de referência foram definidos como um subgrupo de oito dosímetros que tiveram um desvio da resposta menor que $\pm 1,0 \%$ em relação à média de cada série de avaliações realizadas no teste de homogeneidade com todos os setenta e dois dosímetros do grupo.

Os outros sessenta e quatro dosímetros foram escolhidos como dosímetros de campo e utilizados para monitorar a radiação na aplicação dosimétrica que, neste caso, foi a dosimetria clínica. 


\subsubsection{Fator de correção individual}

Algumas variações de sensibilidade dentro de um grupo de dosímetros TL são inevitáveis e vários métodos podem ser utilizados para limitar seus efeitos quando os dosímetros estão em uso comum.

O método utilizado neste trabalho consistiu em tratar termicamente, irradiar com uma dose de $0,5 \mathrm{~Gy}$ todos os dosímetros de referência e de campo do grupo nas mesmas condições geométricas, avaliar todos os dosímetros e atribuir a cada um deles um fator de correção individual $C_{\mathrm{ij}}$ dado por ${ }^{(68)}$ :

$$
C_{i j}=\frac{\bar{R}_{r e f}}{R_{i j}},
$$

onde $\mathrm{R}_{\mathrm{ij}}$ é a leitura TL de cada dosímetro de campo e $\overline{\mathrm{R}}_{\mathrm{ref}}$ é a média da resposta dos dosímetros de referência.

Este procedimento foi repetido quatro vezes e o fator de correção médio de cada dosímetro foi então dado por:

$$
C_{i}=\frac{\sum_{j=1}^{4} C_{i j}}{4},
$$

que expressa a variação da resposta de cada dosímetro individual em torno da média das respostas dos dosímetros de referência. $O$ fator $C_{i}$ foi então associado ao respectivo dosímetro e utilizado como um fator multiplicativo da avaliação para corrigir a resposta do dosímetro irradiado com qualquer dose, isto é:

$$
\mathrm{R}_{\mathrm{i}, \mathrm{corr}}=\mathrm{R}_{\mathrm{i}} \cdot \mathrm{C}_{\mathrm{i}}
$$

\subsubsection{Linearidade}

A ocorrência de não linearidade na curva dose resposta de um detector TL não exclui o seu uso em dosimetria, mas requer uma cuidadosa aplicação de fatores de correção. A curva dose resposta do LiF : Mg, Ti é linear até a dose de $1 \mathrm{~Gy}$, a partir da qual ela se torna supralinear até $10^{3} \mathrm{~Gy}$, onde se tem o início da região de sublinearidade $^{(67)}$.

Na prática, é recomendada a utilização dos dosímetros TL na região em que a resposta é proporcional à dose recebida (região linear), mas quando este não é o caso, uma correção deve ser aplicada ao sinal a partir de uma curva estabelecida com 
o material TL e a leitora usada, e não a partir de uma curva publicada na literatura, pois os parâmetros de leitura podem ter influência na sua forma. Os dosímetros TL não devem ser utilizados na região sublinear ${ }^{(42)}$.

Para determinar a faixa de linearidade do sistema, o grupo de setenta e dois dosímetros de LiF : Mg, Ti foi dividido em nove subgrupos de oito detectores. Para cada um dos subgrupos, irradiados com diferentes doses, foi calculado o valor médio

$$
\overline{\mathrm{R}}=\frac{\sum_{\mathrm{i}} \mathrm{R}_{\mathrm{i}, \text { corr }}}{8},
$$

o que permitiu fazer um gráfico de $\overline{\mathrm{R}}$ em função da dose.

Uma fórmula empírica foi utilizada para corrigir a resposta TL dos dosímetros na região de supralinearidade ${ }^{(45)}$ :

$$
C_{\text {supralin }}=\left(1+0,0278 \cdot D-0,000265 \cdot D^{2}\right)^{-1}
$$

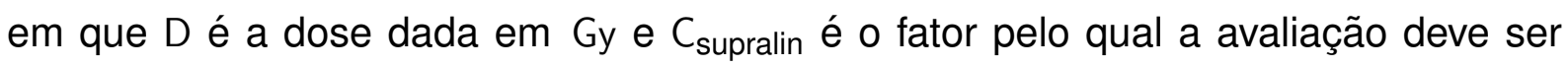
multiplicada para corrigir a supralinearidade. Deve ser notado que D é a dose e não a resposta $T L$, mas $C_{\text {supralin }}$ pode ser calculado iterativamente se $D$ é desconhecida. Esta fórmula é adequada para doses até $20 \mathrm{~Gy}$.

\subsubsection{Calibração}

Nas medições em radioterapia, em que a exatidão na determinação da dose deve estar entre 3-5\%, é imperativo determinar o coeficiente de calibração antes de cada ciclo de avaliação dos dosímetros TL. Neste caso, os dosímetros de referência são irradiados com uma dose apropriada para a calibração e avaliados juntamente com os dosímetros de campo, evitando qualquer efeito de instabilidade da leitora.

Foi determinado um único coeficiente de calibração para todos os dosímetros do grupo utilizando os dosímetros de referência. O coeficiente de calibração para todos os dosímetros do grupo, $N_{D_{w}, \text { grupo }}$, foi determinado antes de cada ciclo de avaliação dos dosímetros TL, utilizando-se a câmara de ionização como instrumento de referência. Os dosímetros TL de referência foram colocados na superfície de entrada do objeto simulador geométrico de acrílico e irradiados no acelerador linear clínico, a uma distância fonte-superfície de $100 \mathrm{~cm}$ e campo de $10 \times 10 \mathrm{~cm}^{2}$, com uma dose de 
0,5 Gy. O coeficiente de calibração para todos os dosímetros do grupo foi dado por:

$$
\mathrm{N}_{\mathrm{D}_{\mathrm{w}, \text { grupo }}}=\frac{1}{8} \sum_{\mathrm{i}} \frac{\mathrm{D}_{\mathrm{i}}}{\mathrm{R}_{\mathrm{i}} \cdot \mathrm{C}_{\mathrm{i}}}
$$

em que $D_{i}$ é a dose absorvida na água, medida pela câmara de ionização seguindo o protocolo TRS 398 da International Atomic Energy Agency ${ }^{(83)}, \mathrm{R}_{\mathrm{i}}$ é resposta TL do i-ésimo dosímetro de referência e $C_{i}$ é o fator de correção individual associado.

Os sessenta e quatro dosímetros de campo foram utilizados para realizar as medições de dose dadas por:

$$
\mathrm{D}=\mathrm{R}_{\mathrm{i}} \cdot \mathrm{N}_{\mathrm{D}_{\mathrm{w}, \text { grupo }}} \cdot \mathrm{C}_{\mathrm{i}} \cdot \mathrm{C}_{\text {supralin }} \cdot \mathrm{C}_{\text {energia }}
$$

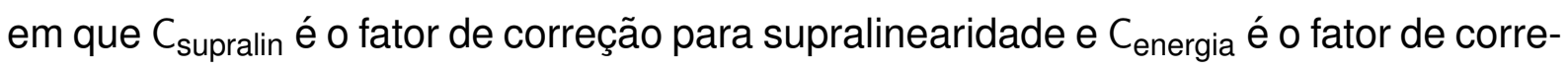
ção para dependência energética que, neste caso, é igual a um, já que os dosímetros TL de referência foram calibrados em relação à câmara de ionização utilizando a qualidade do feixe que foi usada para as medições com os dosímetros TL de campo ${ }^{(42,45)}$.

\subsection{Medições no objeto simulador antropomórfico}

Para verificação do sistema dosimétrico, foram usadas as condições normais de tratamento de câncer na região pélvica em um objeto simulador antropomórfico Alderson Rando no formato de um homem adulto sem os membros $(1,73 \mathrm{~m}$ da cabeça ao torso e $73,5 \mathrm{Kg}$ ). O planejamento envolveu uma tomografia computadorizada do objeto simulador antropomórfico para o cálculo de dose utilizando o sistema InterSoftC. O objeto simulador antropomórfico foi irradiado utilizando-se campos látero-laterais diretos e anterior.

Para cada irradiação foi usado um único dosímetro que, em conjunto com uma espessura de equilíbrio eletrônico de latão, foi fixado com esparadrapo sobre a marcação do ponto de incidência do feixe de radiação previamente feita sobre o objeto simulador antropomórfico; tomou-se o cuidado de manter a superfície da espessura da latão perpendicular ao feixe incidente. 


\subsection{Medições in vivo}

Confirmada a validade do sistema dosimétrico estabelecido e verificada a possibilidade de sua aplicação in vivo para a medições de doses de entrada, o próximo passo foi obter a permissão de um dos pacientes do Serviço de Radioterapia do HCFMRP (por meio de um termo de consentimento livre e esclarecido) para o uso dos dosímetros TL em algumas das sessões de tratamento para a comparação das doses previstas no sistema de planejamento de tratamento para alguns dos campos com as doses obtidas pelo sistema dosimétrico.

Foram acompanhadas 5 sessões de um tratamento de câncer de próstata que utilizava cinco campos:

- Campo nำ 1: oblíquo posterior direito;

- Campo no 2: oblíquo anterior direito;

- Campo no 3: anterior;

- Campo no 4: oblíquo anterior esquerdo;

- Campo n 5: oblíquo posterior esquerdo.

Para as medições em cada um dos campos, foi usado um único dosímetro que, em conjunto com a espessura de equilíbrio eletrônico de latão, foi fixado com esparadrapo hipoalergênico sobre a marcação do ponto de incidência do feixe de radiação, feita previamente sobre a pele do paciente, tomando-se novamente o cuidado de manter a superfície da espessura de latão perpendicular ao feixe incidente. 


\section{$3 \quad$ Resultados e Discussões}

\subsection{Estabelecimento do sistema dosimétrico TL}

\subsubsection{Procedimento de inicialização}

Como já foi dito, o procedimento de inicialização envolveu o tratamento térmico dos dosímetros utilizando os parâmetros indicados para o LiF : Mg, Ti e sua avaliação para verificação do sinal de fundo. Estes procedimentos foram repetidos em mais dois ciclos. Como os sinais de fundo dos dosímetros permaneceram baixos durante estes ciclos, a inicialização foi terminada e os dosímetros foram submetidos aos testes subseqüentes. A Tabela 3.1 mostra os resultados obtidos.

Tabela 3.1: Procedimento de inicialização. $R_{\mathrm{ij0}}$ é o sinal de fundo e CV é o desvio padrão porcentual.

\begin{tabular}{cccccc}
\hline Ciclo j & 1 & 2 & 3 & & \\
\hline Dosímetro i & $\mathrm{R}_{\mathrm{ij} 0}(\mathrm{nC})$ & $\mathrm{R}_{\mathrm{ij} 0}(\mathrm{nC})$ & $\mathrm{R}_{\mathrm{ij0}}(\mathrm{nC})$ & $\overline{\mathrm{R}}_{\mathrm{ij} 0}(\mathrm{nC})$ & $\mathrm{CV}(\%)$ \\
\hline 1 & 0,222 & 0,180 & 0,113 & & \\
2 & 0,186 & 0,220 & 0,059 & & \\
3 & 0,147 & 0,159 & 0,118 & & \\
4 & 0,286 & 0,094 & 0,134 & & \\
6 & 0,226 & 0,109 & 0,127 & & \\
7 & 0,132 & 0,171 & 0,097 & & \\
8 & 0,133 & 0,125 & 0,077 & \\
9 & 0,193 & 0,129 & 0,115 & \\
10 & 0,185 & 0,101 & 0,110 & \\
11 & 0,092 & 0,126 & 0,080 & \\
12 & 0,141 & 0,140 & 0,118 &
\end{tabular}


continuação

\begin{tabular}{|c|c|c|c|c|c|}
\hline Ciclo j & 1 & 2 & 3 & & \\
\hline Dosímetro i & $\mathrm{R}_{\mathrm{ij} 0}(\mathrm{nC})$ & $\mathrm{R}_{\mathrm{ij} 0}(\mathrm{nC})$ & $\mathrm{R}_{\mathrm{ij} 0}(\mathrm{nC})$ & $\overline{\overline{\mathrm{R}}}_{\mathrm{ij} 0}(\mathrm{nC})$ & CV (\%) \\
\hline 13 & 0,154 & 0,104 & 0,082 & & \\
\hline 14 & 0,126 & 0,141 & 0,118 & & \\
\hline 15 & 0,144 & 0,128 & 0,120 & & \\
\hline 16 & 0,151 & 0,124 & 0,131 & & \\
\hline 17 & 0,132 & 0,161 & 0,072 & & \\
\hline 18 & 0,115 & 0,164 & 0,121 & & \\
\hline 19 & 0,120 & 0,129 & 0,070 & & \\
\hline 20 & 0,142 & 0,082 & 0,081 & & \\
\hline 21 & 0,111 & 0,079 & 0,096 & & \\
\hline 22 & 0,117 & 0,235 & 0,089 & & \\
\hline 23 & 0,165 & 0,155 & 0,141 & & \\
\hline 24 & 0,095 & 0,094 & 0,089 & & \\
\hline 25 & 0,143 & 0,128 & 0,101 & & \\
\hline 26 & 0,047 & 0,162 & 0,159 & & \\
\hline 27 & 0,204 & 0,159 & 0,050 & & \\
\hline 28 & 0,157 & 0,127 & 0,092 & & \\
\hline 29 & 0,164 & 0,169 & 0,088 & & \\
\hline 30 & 0,137 & 0,119 & 0,083 & & \\
\hline 31 & 0,113 & 0,172 & 0,073 & & \\
\hline 32 & 0,117 & 0,126 & 0,070 & & \\
\hline 33 & 0,133 & 0,139 & 0,088 & & \\
\hline 34 & 0,158 & 0,105 & 0,081 & & \\
\hline 35 & 0,125 & 0,141 & 0,117 & & \\
\hline 36 & 0,135 & 0,117 & 0,099 & & \\
\hline 37 & 0,158 & 0,159 & 0,124 & & \\
\hline 38 & 0,118 & 0,068 & 0,113 & & \\
\hline 39 & 0,166 & 0,102 & 0,099 & & \\
\hline 40 & 0,110 & 0,098 & 0,129 & & \\
\hline 41 & 0,105 & 0,101 & 0,091 & & \\
\hline 43 & 0,135 & 0,099 & 0,113 & & \\
\hline 44 & 0,120 & 0,135 & 0,066 & & \\
\hline 45 & 0,174 & 0,129 & 0,079 & & \\
\hline
\end{tabular}


continuação

\begin{tabular}{|c|c|c|c|c|c|}
\hline Ciclo j & 1 & 2 & 3 & & \\
\hline Dosímetro i & $\mathrm{R}_{\mathrm{ij} 0}(\mathrm{nC})$ & $\mathrm{R}_{\mathrm{ij} 0}(\mathrm{nC})$ & $\mathrm{R}_{\mathrm{ij} 0}(\mathrm{nC})$ & $\overline{\overline{\mathrm{R}}}_{\mathrm{ij} 0}(\mathrm{nC})$ & CV (\%) \\
\hline 46 & 0,170 & 0,122 & 0,095 & & \\
\hline 47 & 0,166 & 0,159 & 0,075 & & \\
\hline 48 & 0,198 & 0,121 & 0,096 & & \\
\hline 49 & 0,157 & 0,116 & 0,086 & & \\
\hline 50 & 0,134 & 0,110 & 0,119 & & \\
\hline 51 & 0,107 & 0,081 & 0,087 & & \\
\hline 52 & 0,102 & 0,094 & 0,083 & & \\
\hline 53 & 0,185 & 0,126 & 0,135 & & \\
\hline 54 & 0,093 & 0,094 & 0,122 & & \\
\hline 55 & 0,106 & 0,086 & 0,094 & & \\
\hline 57 & 0,202 & 0,112 & 0,059 & & \\
\hline 58 & 0,154 & 0,139 & 0,100 & & \\
\hline 59 & 0,132 & 0,091 & 0,163 & & \\
\hline 60 & 0,137 & 0,082 & 0,127 & & \\
\hline 61 & 0,107 & 0,103 & 0,150 & & \\
\hline 62 & 0,112 & 0,092 & 0,110 & & \\
\hline 63 & 0,132 & 0,070 & 0,135 & & \\
\hline 64 & 0,122 & 0,124 & 0,088 & & \\
\hline 65 & 0,098 & 0,095 & 0,081 & & \\
\hline 66 & 0,097 & 0,091 & 0,110 & & \\
\hline 67 & 0,135 & 0,085 & 0,204 & & \\
\hline 68 & 0,145 & 0,119 & 0,102 & & \\
\hline 69 & 0,164 & 0,162 & 0,232 & & \\
\hline 70 & 0,128 & 0,141 & 0,139 & & \\
\hline 71 & 0,093 & 0,138 & 0,126 & & \\
\hline 72 & 0,103 & 0,082 & 0,045 & & \\
\hline 73 & 0,102 & 0,108 & 0,131 & & \\
\hline 74 & 0,097 & 0,096 & 0,121 & & \\
\hline 75 & 0,101 & 0,089 & 0,092 & & \\
\hline$\overline{\mathrm{R}}_{\mathrm{ij} 0}$ & 0,139 & 0,123 & 0,105 & 0,122 & 14 \\
\hline $\mathrm{CV}(\%)$ & 27 & 30 & 30 & & \\
\hline
\end{tabular}


Apesar dos valores altos obtidos para os desvios padrão porcentuais dos $\mathrm{R}_{\mathrm{ij} 0} \mathrm{e}$ dos $\bar{R}_{i j 0}$, a intenção do procedimento de inicialização foi reduzir a possibilidade de futuras mudanças de características e de resposta dos TLDs durante seu uso, o que poderia levar a variações indesejadas nos resultados obtidos, e sua avaliação não é possível somente a partir da observação dos dados da Tabela 3.1, já que a existência de armadilhas ainda não preenchidas permanentemente nos dosímetros lhes confere uma resposta ainda instável, apesar da aparente tendência de diminuição dos valores de $\overline{\mathrm{R}}_{\mathrm{ij} 0}$. Além do mais, é necessário lembrar a modalidade à qual se propõe o uso destes detectores, que prevê a medida de doses superiores a $1 \mathrm{~Gy}$, o que reduz a relevância das flutuações de seus valores de fundo relativamente baixos.

\subsubsection{Homogeneidade do grupo}

Após a pré-irradiação e a divisão dos dosímetros em um grupo de referência e um de campo, os dosímetros TL foram colocados em um suporte de acrílico, irradiados com uma dose de 0,5 Gy juntamente com a espessura de equilíbrio eletrônico, avaliados e termicamente tratados, o que se repetiu mais três vezes. Os resultados obtidos estão apresentado na Tabela 3.2 (as células em branco se referem aos dosímetros que, devido ao difícil manuseio, foram perdidos).

Tabela 3.2: Procedimento para o teste de homogeneidade do grupo de dosímetros. $\mathrm{R}_{\mathrm{ij}}$ é a resposta $T L$ de cada dosímetro e os números marcados um $\left(^{*}\right)$ se referem aos dosímetros de referência.

\begin{tabular}{ccccc}
\hline Irradiação j & 1 & 2 & 3 & 4 \\
\hline Dosímetro i & $\mathrm{R}_{\mathrm{ij}}(\mathrm{nC})$ & $\mathrm{R}_{\mathrm{ij}}(\mathrm{nC})$ & $\mathrm{R}_{\mathrm{ij}}(\mathrm{nC})$ & $\mathrm{R}_{\mathrm{ij}}(\mathrm{nC})$ \\
\hline 1 & 795 & 778,7 & 823,8 & 723 \\
2 & 832,2 & 833 & 877,5 & 785,6 \\
3 & 824,3 & 821,3 & 846,8 & 752,7 \\
4 & 889,9 & 864,5 & 902,7 & 788,2 \\
5 & & & & \\
6 & 793,9 & 801,5 & 824,8 & 716,1 \\
\multicolumn{5}{c}{ continua } \\
\end{tabular}


continuação

\begin{tabular}{ccccc}
\hline Irradiação j & 1 & 2 & 3 & 4 \\
\hline Dosímetro i & $\mathrm{R}_{\mathrm{ij}}(\mathrm{nC})$ & $\mathrm{R}_{\mathrm{ij}}(\mathrm{nC})$ & $\mathrm{R}_{\mathrm{ij}}(\mathrm{nC})$ & $\mathrm{R}_{\mathrm{ij}}(\mathrm{nC})$ \\
\hline 7 & 872,3 & 842,2 & 892,4 & 753,7 \\
8 & 793,8 & 785,5 & 823,2 & 690,3 \\
9 & 874,2 & 851,5 & 912,2 & 754,2 \\
10 & 820,2 & 799 & 848,2 & 696,3 \\
11 & 825,9 & 794,7 & 873,4 & \\
12 & 851,5 & 817,7 & 809,8 & 765,6 \\
13 & 901,7 & 864,1 & 876,6 & 792,7 \\
14 & 848,1 & 786,1 & 794,6 & 729,9 \\
15 & 891,8 & 816,5 & 869,1 & 758,1 \\
16 & 847,6 & 816,9 & 860,9 & 724 \\
17 & 825,5 & 779,7 & 828,9 & 691,3 \\
18 & 891,9 & 836,6 & 824,6 & 739,2 \\
19 & 869,2 & 815,6 & 842,8 & 733,5 \\
20 & 828,4 & 790,9 & 810,6 & 702,4 \\
$21^{*}$ & 835,2 & 793,5 & 816,6 & 750,5 \\
22 & 831,1 & 792,4 & 799,6 & 740,4 \\
23 & 801,4 & 757,3 & 784 & 695,8 \\
24 & 885,4 & 828,5 & 830,2 & 762,6 \\
25 & 889,6 & 833 & 827,8 & 764,2 \\
26 & 870,5 & 823,7 & 831,6 & 737 \\
27 & 855,3 & 787,7 & 795,8 & 715,2 \\
28 & 852,9 & 806 & 793,3 & 718,2 \\
29 & 922,7 & 875 & 875,9 & 782,6 \\
30 & 827,5 & 781,6 & 809,5 & 702 \\
31 & 830,5 & 786,1 & 871,4 & 754,8 \\
32 & 844 & 807 & 867,3 & 761,5 \\
33 & 782,3 & 730,4 & 757,2 & 688,5 \\
34 & 820,4 & 773,9 & 779 & 727,6 \\
35 & 785,5 & 723,1 & 754,2 & 682,3 \\
36 & 841,2 & 770,6 & 808,9 & 732,5 \\
37 & 864 & 793,1 & 844,2 & 740,3 \\
38 & 895,5 & 828,3 & 862,1 & 766,1 \\
& & $\mathrm{continua}$ & & \\
& & & &
\end{tabular}


continuação

\begin{tabular}{|c|c|c|c|c|}
\hline Irradiação j & 1 & 2 & 3 & 4 \\
\hline Dosímetro i & $\mathrm{R}_{\mathrm{ij}}(\mathrm{nC})$ & $\mathrm{R}_{\mathrm{ij}}(\mathrm{nC})$ & $\mathrm{R}_{\mathrm{ij}}(\mathrm{nC})$ & $\mathrm{R}_{\mathrm{ij}}(\mathrm{nC})$ \\
\hline 39 & 833,8 & 782,1 & 811 & 717,6 \\
\hline 40 & 870,2 & 807,7 & 906,1 & 760,3 \\
\hline $41^{*}$ & 812 & 765,7 & 861,1 & 737,4 \\
\hline \multicolumn{5}{|l|}{42} \\
\hline $43^{*}$ & 822,9 & 757,8 & 849,8 & 748,2 \\
\hline 44 & 856,3 & 781,9 & 873,4 & 755 \\
\hline $45^{*}$ & 833,8 & 791,2 & 831,1 & 748,7 \\
\hline 46 & 817,9 & 759,9 & 825,4 & 712,7 \\
\hline 47 & 819,2 & 763 & 792,9 & 718,1 \\
\hline 48 & 867,1 & 796,9 & 887,6 & 764,5 \\
\hline 49 & 872,6 & 813,5 & 893,5 & 765,2 \\
\hline 50 & 800 & 734,9 & 824,7 & 714,5 \\
\hline 51 & 825,1 & 773,9 & 848,9 & 786,5 \\
\hline 52 & 848,5 & 805,9 & 842,2 & 786,1 \\
\hline 53 & 877,5 & 809,1 & 909,2 & 816,5 \\
\hline 54 & 823,6 & 760,7 & 802,6 & 756 \\
\hline 55 & 804,5 & 739,7 & 803,6 & 739,7 \\
\hline \multicolumn{5}{|l|}{56} \\
\hline $57^{*}$ & 825,4 & 787,8 & 841,1 & 753,6 \\
\hline $58^{*}$ & 822,1 & 764,8 & 866,8 & 747,9 \\
\hline 59 & 830,1 & 753,6 & 840,6 & 732,2 \\
\hline 60 & 805,9 & 762,8 & 814,2 & 722,2 \\
\hline 61 & 795,4 & 763,7 & 831,7 & 749,9 \\
\hline 62 & 835,6 & 789,8 & 829,2 & 793,4 \\
\hline $63^{*}$ & 842,6 & 763,3 & 817,5 & 780,9 \\
\hline 64 & 800,2 & 748,6 & 831 & 748,8 \\
\hline 65 & 755,3 & 702,2 & 779,7 & 713,4 \\
\hline 66 & 762,9 & 708 & 811,8 & 716,7 \\
\hline 67 & 814,1 & 739,3 & 841 & 748,2 \\
\hline 68 & 848,2 & 797,9 & 903,8 & 789,3 \\
\hline $69^{*}$ & 839,3 & 777,3 & 830,8 & 762,9 \\
\hline 70 & 858,6 & 807,1 & 916,9 & 782,1 \\
\hline
\end{tabular}


continuação

\begin{tabular}{ccccc}
\hline Irradiação j & 1 & 2 & 3 & 4 \\
\hline Dosímetro i & $\mathrm{R}_{\mathrm{ij}}(\mathrm{nC})$ & $\mathrm{R}_{\mathrm{ij}}(\mathrm{nC})$ & $\mathrm{R}_{\mathrm{ij}}(\mathrm{nC})$ & $\mathrm{R}_{\mathrm{ij}}(\mathrm{nC})$ \\
\hline 71 & 819,5 & 801,9 & 853 & 789,9 \\
72 & 741 & 683,8 & 741,2 & 710,6 \\
73 & 766,8 & 712,3 & 809,5 & 736,7 \\
74 & 779,2 & 726,5 & 814,6 & 754,7 \\
75 & 778 & 717 & 800,3 & 742,5 \\
\hline$\Delta_{\max }$ & 24,52 & 27,96 & 23,70 & 19,67 \\
\hline
\end{tabular}

Com os dados da Tabela 3.2 pode-se calcular quatro diferentes valores para 0 índice de homogeneidade, $\Delta_{\max }$. Os valores obtidos foram menores que $30 \mathrm{em}$ todos os casos o que permitiu que todo o grupo fosse utilizado sem o descarte de nenhum dosímetro.

\subsubsection{Fator de correção individual}

Com os valores de $\mathrm{R}_{\mathrm{ij}}$ dos dosímetros de referência, apresentados na Tabela 3.2, calculou-se o valor do fator de correção individual, $C_{i j}$, e a partir destes, determinou-se o fator de correção individual médio, $C_{i}$, e a respectiva incerteza relativa, $\sigma_{i} / C_{i}$, para cada um dos dosímetros (Tabela 3.3).

Tabela 3.3: Fator de correção individual para cada dosímetro. $\sigma_{\mathrm{i}} / \mathrm{C}_{\mathrm{i}}$ é a incerteza relativa no fator de correção individual médio, $\mathrm{C}_{\mathrm{i}}$.

\begin{tabular}{ccccccc}
\hline Dosímetro i & $C_{i j}$ & $C_{i j}$ & $C_{i j}$ & $C_{i j}$ & $C_{i}$ & $\sigma_{i} / C_{i}$ \\
\hline 1 & 1,036 & 0,996 & 1,021 & 1,037 & 1,022 & 0,009 \\
2 & 0,989 & 0,931 & 0,959 & 0,955 & 0,958 & 0,013 \\
3 & 0,999 & 0,944 & 0,993 & 0,996 & 0,983 & 0,013 \\
4 & 0,925 & 0,897 & 0,932 & 0,951 & 0,926 & 0,012 \\
5 & & & & & & \\
6 & 1,037 & 0,967 & 1,020 & 1,047 & 1,018 & 0,017 \\
& \multicolumn{6}{c}{ continua } \\
& \multicolumn{6}{c}{}
\end{tabular}


continuação

\begin{tabular}{ccccccc}
\hline Dosímetro i & $C_{\mathrm{ij}}$ & $\mathrm{C}_{\mathrm{ij}}$ & $\mathrm{C}_{\mathrm{ij}}$ & $\mathrm{C}_{\mathrm{ij}}$ & $\mathrm{C}_{\mathrm{i}}$ & $\sigma_{\mathrm{i}} / \mathrm{C}_{\mathrm{i}}$ \\
\hline 7 & 0,944 & 0,920 & 0,943 & 0,995 & 0,950 & 0,017 \\
8 & 1,037 & 0,987 & 1,022 & 1,086 & 1,033 & 0,020 \\
9 & 0,942 & 0,910 & 0,922 & 0,994 & 0,942 & 0,020 \\
10 & 1,004 & 0,970 & 0,992 & 1,077 & 1,011 & 0,023 \\
11 & 0,997 & 0,975 & 0,963 & & 0,978 & 0,010 \\
12 & 0,967 & 0,948 & 1,039 & 0,979 & 0,983 & 0,020 \\
13 & 0,913 & 0,897 & 0,960 & 0,946 & 0,929 & 0,016 \\
14 & 0,971 & 0,986 & 1,059 & 1,027 & 1,011 & 0,020 \\
15 & 0,923 & 0,949 & 0,968 & 0,989 & 0,957 & 0,015 \\
16 & 0,971 & 0,949 & 0,977 & 1,036 & 0,983 & 0,019 \\
17 & 0,997 & 0,994 & 1,015 & 1,085 & 1,023 & 0,021 \\
18 & 0,923 & 0,927 & 1,020 & 1,014 & 0,971 & 0,028 \\
19 & 0,947 & 0,950 & 0,998 & 1,022 & 0,980 & 0,019 \\
20 & 0,994 & 0,980 & 1,038 & 1,068 & 1,020 & 0,020 \\
21 & 0,986 & 0,977 & 1,030 & 0,999 & 0,998 & 0,012 \\
22 & 0,991 & 0,978 & 1,052 & 1,013 & 1,008 & 0,016 \\
23 & 1,027 & 1,024 & 1,073 & 1,078 & 1,050 & 0,014 \\
24 & 0,930 & 0,936 & 1,013 & 0,983 & 0,966 & 0,021 \\
25 & 0,925 & 0,931 & 1,016 & 0,981 & 0,963 & 0,022 \\
26 & 0,946 & 0,941 & 1,011 & 1,017 & 0,979 & 0,021 \\
27 & 0,963 & 0,984 & 1,057 & 1,049 & 1,013 & 0,023 \\
28 & 0,965 & 0,962 & 1,060 & 1,044 & 1,008 & 0,026 \\
29 & 0,892 & 0,886 & 0,960 & 0,958 & 0,924 & 0,022 \\
30 & 0,995 & 0,992 & 1,039 & 1,068 & 1,024 & 0,018 \\
31 & 0,991 & 0,986 & 0,965 & 0,993 & 0,984 & 0,007 \\
32 & 0,975 & 0,961 & 0,970 & 0,985 & 0,973 & 0,005 \\
33 & 1,052 & 1,061 & 1,111 & 1,089 & 1,078 & 0,012 \\
34 & 1,003 & 1,002 & 1,080 & 1,031 & 1,029 & 0,018 \\
35 & 1,048 & 1,072 & 1,115 & 1,099 & 1,084 & 0,014 \\
36 & 0,979 & 1,006 & 1,040 & 1,024 & 1,012 & 0,013 \\
37 & 0,953 & 0,977 & 0,996 & 1,013 & 0,985 & 0,013 \\
38 & 0,919 & 0,936 & 0,976 & 0,979 & 0,952 & 0,015 \\
39 & 0,987 & 0,991 & 1,037 & 1,045 & 1,015 & 0,015 \\
& & continua & & & \\
& & & & & &
\end{tabular}


continuação

\begin{tabular}{ccccccc}
\hline Dosímetro i & $C_{i j}$ & $C_{i j}$ & $C_{i j}$ & $C_{i j}$ & $C_{i}$ & $\sigma_{i} / C_{i}$ \\
\hline 40 & 0,946 & 0,960 & 0,928 & 0,986 & 0,955 & 0,013 \\
41 & 1,014 & 1,012 & 0,977 & 1,017 & 1,005 & 0,009 \\
42 & & & & & & \\
43 & 1,000 & 1,023 & 0,990 & 1,002 & 1,004 & 0,007 \\
44 & 0,961 & 0,991 & 0,963 & 0,993 & 0,977 & 0,009 \\
45 & 0,987 & 0,980 & 1,012 & 1,002 & 0,995 & 0,007 \\
46 & 1,007 & 1,020 & 1,019 & 1,052 & 1,024 & 0,009 \\
47 & 1,005 & 1,016 & 1,061 & 1,044 & 1,032 & 0,012 \\
48 & 0,949 & 0,973 & 0,948 & 0,981 & 0,963 & 0,009 \\
49 & 0,943 & 0,953 & 0,941 & 0,980 & 0,954 & 0,009 \\
50 & 1,029 & 1,055 & 1,020 & 1,050 & 1,038 & 0,008 \\
51 & 0,998 & 1,002 & 0,991 & 0,953 & 0,986 & 0,011 \\
52 & 0,970 & 0,962 & 0,999 & 0,954 & 0,971 & 0,010 \\
53 & 0,938 & 0,958 & 0,925 & 0,918 & 0,935 & 0,009 \\
54 & 1,000 & 1,019 & 1,048 & 0,992 & 1,015 & 0,012 \\
55 & 1,023 & 1,048 & 1,047 & 1,014 & 1,033 & 0,008 \\
56 & & & & & & \\
57 & 0,997 & 0,984 & 1,000 & 0,995 & 0,994 & 0,004 \\
58 & 1,001 & 1,014 & 0,970 & 1,003 & 0,997 & 0,009 \\
59 & 0,992 & 1,029 & 1,001 & 1,024 & 1,011 & 0,009 \\
60 & 1,022 & 1,016 & 1,033 & 1,038 & 1,027 & 0,005 \\
61 & 1,035 & 1,015 & 1,011 & 1,000 & 1,015 & 0,007 \\
62 & 0,985 & 0,982 & 1,014 & 0,945 & 0,982 & 0,014 \\
63 & 0,977 & 1,016 & 1,029 & 0,960 & 0,995 & 0,016 \\
64 & 1,029 & 1,036 & 1,012 & 1,001 & 1,020 & 0,008 \\
65 & 1,090 & 1,104 & 1,079 & 1,051 & 1,081 & 0,010 \\
66 & 1,079 & 1,095 & 1,036 & 1,046 & 1,064 & 0,013 \\
67 & 1,011 & 1,049 & 1,000 & 1,002 & 1,016 & 0,011 \\
68 & 0,971 & 0,972 & 0,931 & 0,950 & 0,956 & 0,010 \\
69 & 0,981 & 0,997 & 1,012 & 0,983 & 0,993 & 0,007 \\
70 & 0,959 & 0,961 & 0,917 & 0,959 & 0,949 & 0,011 \\
71 & 1,005 & 0,967 & 0,986 & 0,949 & 0,977 & 0,012 \\
72 & 1,111 & 1,134 & 1,135 & 1,055 & 1,109 & 0,017 \\
& & $c 0 n t i n u a$ & & &
\end{tabular}


continuação

\begin{tabular}{ccccccc}
\hline Dosímetro i & $C_{i j}$ & $C_{i j}$ & $C_{i j}$ & $C_{i j}$ & $C_{i}$ & $\sigma_{i} / C_{i}$ \\
\hline 73 & 1,074 & 1,088 & 1,039 & 1,018 & 1,055 & 0,015 \\
74 & 1,057 & 1,067 & 1,033 & 0,994 & 1,037 & 0,016 \\
75 & 1,058 & 1,081 & 1,051 & 1,010 & 1,050 & 0,014 \\
\hline
\end{tabular}

\subsubsection{Linearidade}

A Figura 3.1 mostra a curva da reposta TL em função da dose para verificação da linearidade da resposta dos dosímetros TL no intervalo de dose em que, neste caso, propõe-se a dosimetria in vivo.

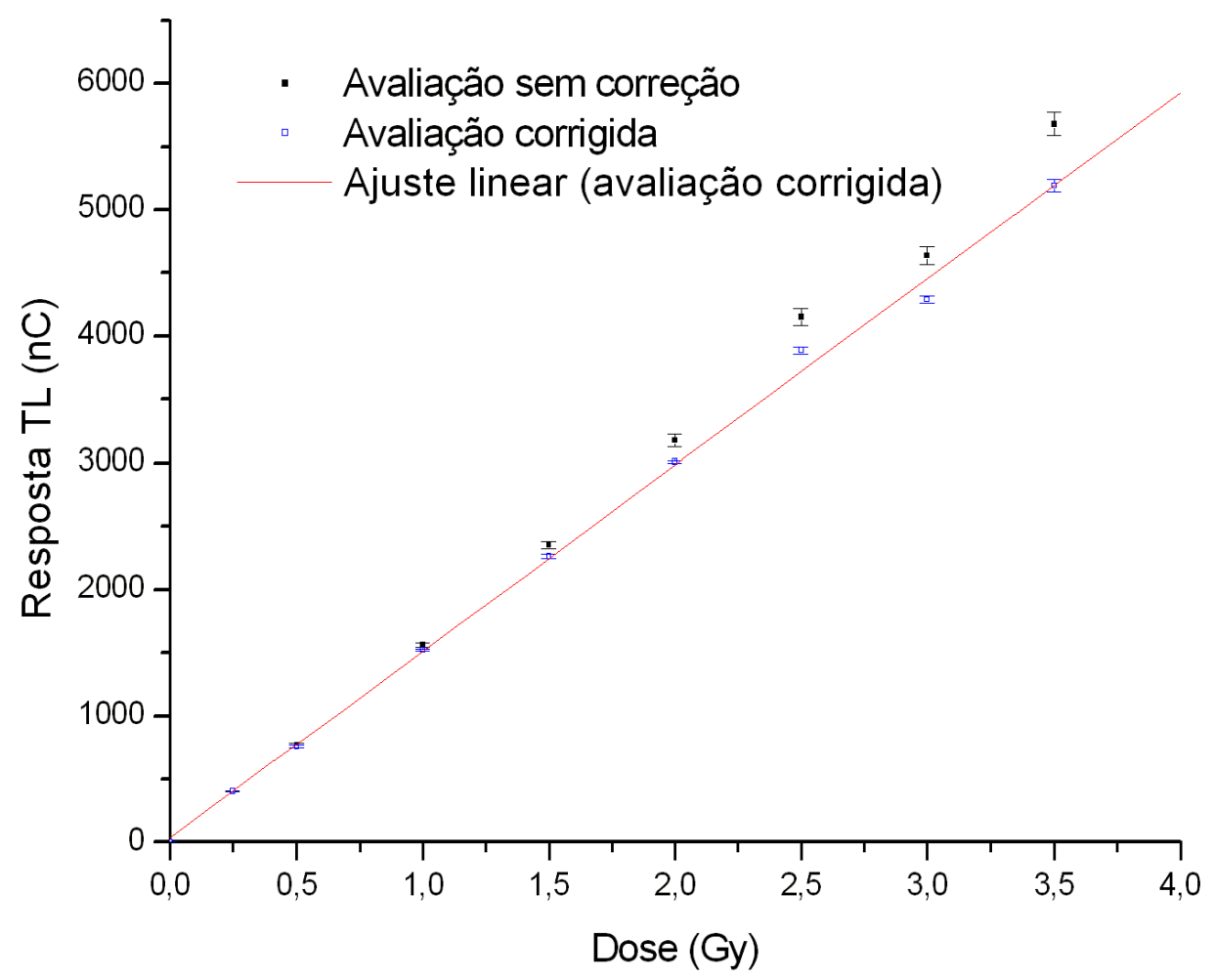

Figura 3.1: Resposta TL em função da dose.

Faz-se importante observar que a fórmula empírica para a correção da resposta TL dos dosímetros foi aplicada a todos os valores de $\bar{R}$ obtidos, independentemente 
de pertencerem ou não à região de supralinearidade; como resultado, é possível observar na Figura 3.1 uma tendência de aumento das respostas TL não corrigidas dos dosímetros submetidos a doses de valores mais altos, em comparação com os valores corrigidos pela fórmula, o que corrobora as informações fornecidas pela literatura e indica a validade dos procedimentos até então utilizados. Quanto ao resultado da correção, a curva de regressão linear se ajustou bem aos valores de resposta corrigidos.

\subsection{Medições no objeto simulador antropomórfico}

O primeiro passo para a realização das medições de dose no objeto simulador antropomórfico foi a determinação do coeficiente de calibração para todos os dosímetros

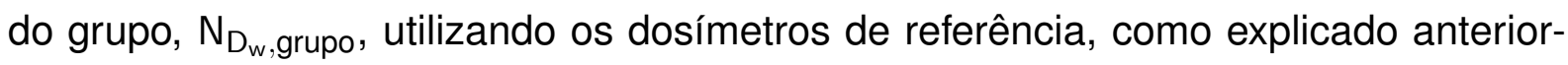
mente.

A Tabela 3.4 apresenta os resultados de dose de entrada obtidos para as irradiações do objeto simulador antropomórfico, bem como sua comparação com os valores previstos pelo sistema de planejamento do tratamento.

Tabela 3.4: Resultados obtidos nas medições de dose no objeto simulador antropomórfico.

\begin{tabular}{ccccc}
\hline Campo & $\begin{array}{c}\text { Dose medida } \\
(\text { Gy })\end{array}$ & $\begin{array}{c}\text { Incerteza relativa } \\
(\%)\end{array}$ & $\begin{array}{c}\text { Dose prescrita } \\
(\text { Gy })\end{array}$ & $\begin{array}{c}\text { Desvio } \\
(\%)\end{array}$ \\
\hline Antero- & 1,346 & 1,7 & 1,290 & 4,3 \\
posterior & 1,290 & 1,9 & 1,290 & 0,0 \\
& 1,294 & 2,2 & 1,290 & 0,4 \\
\hline Lateral & 1,611 & 2,4 & 1,580 & 2,0 \\
Direito & 1,600 & 2,7 & 1,580 & 1,3 \\
& 1,542 & 2,4 & 1,580 & $-2,4$ \\
\hline
\end{tabular}

Obteve-se um desvio máximo de 4,3\% entre a dose medida e a dose prescrita. Levando-se em consideração as incertezas nas medições de dose, estes resultados 
foram considerados excelentes, garantindo que a metodologia é adequada e pode ser utilizada para medições in vivo.

\subsection{Medições in vivo}

A Tabela 3.5 apresenta os resultados de dose obtidos para as medições in vivo, bem como a comparação com os valores previstos pelo sistema de planejamento do tratamento para os cinco campos planejados:

- Campo no 1: oblíquo posterior direito;

- Campo no 2: oblíquo anterior direito;

- Campo no 3: anterior;

- Campo no 4: oblíquo anterior esquerdo;

- Campo no 5: oblíquo posterior esquerdo.

Tabela 3.5: Resultados obtidos nas medições de dose in vivo.

Campo Dose medida Incerteza relativa Dose prescrita Desvio

\begin{tabular}{ccccc} 
& $($ Gy $)$ & $(\%)$ & $($ Gy $)$ & $(\%)$ \\
\hline 1 & 0,889 & 1,9 & 0,920 & $-3,4$ \\
2 & 0,594 & 1,4 & 0,690 & $-13,9$ \\
3 & 0,537 & 1,2 & 0,520 & 3,3 \\
4 & 0,540 & 1,5 & 0,650 & $-17,0$ \\
5 & 0,850 & 1,5 & 0,940 & $-9,5$ \\
\hline 1 & 0,877 & 2,1 & 0,920 & $-4,7$ \\
2 & 0,606 & 1,7 & 0,690 & $-12,2$ \\
3 & 0,540 & 2,4 & 0,520 & 3,8 \\
4 & 0,549 & 2,1 & 0,650 & $-15,5$ \\
5 & 0,837 & 1,3 & 0,940 & $-11,0$ \\
\hline 1 & 0,912 & 2,2 & 0,920 & $-0,9$ \\
2 & 0,607 & 2,4 & 0,690 & $-12,0$ \\
3 & 0,544 & 1,8 & 0,520 & 4,7
\end{tabular}


continuação

Campo Dose medida Incerteza relativa Dose prescrita Desvio

\begin{tabular}{ccccc} 
& $($ Gy $)$ & $(\%)$ & $($ Gy $)$ & $(\%)$ \\
\hline 4 & 0,555 & 2,2 & 0,650 & $-14,5$ \\
5 & 0,864 & 1,6 & 0,940 & $-8,1$ \\
\hline 1 & 0,916 & 1,0 & 0,920 & $-0,4$ \\
2 & 0,593 & 0,8 & 0,690 & $-14,0$ \\
3 & 0,565 & 2,4 & 0,520 & 8,6 \\
4 & 0,566 & 2,3 & 0,650 & $-12,9$ \\
5 & 0,893 & 2,7 & 0,940 & $-5,0$ \\
\hline 1 & 0,942 & 1,5 & 0,920 & 2,4 \\
2 & 0,690 & 1,6 & 0,690 & 0,0 \\
3 & 0,548 & 0,9 & 0,520 & 5,4 \\
4 & 0,578 & 1,9 & 0,650 & $-11,1$ \\
5 & 0,920 & 1,5 & 0,940 & $-2,1$ \\
\hline
\end{tabular}

Os grandes desvios entre os valores de dose medidos e os valores previstos para os campos 2 e 4, apesar de terem sido previstos, não foram descartados justamente para verificar uma das importantes característica dos dosímetros TL e da metodologia envolvida em sua utilização.

Apesar do fato de que os campos 1, 2, 4 e 5 serem todos oblíquos, os campos 1 e 5 formam um ângulo de apenas $5^{\circ}$ com a normal à superfície do paciente no ponto de incidência do feixe de radiação, ao passo que os campos 2 e 4 formam um ângulo de $45^{\circ}$. Sabe-se que os dosímetros TL não apresentam dependência angular, porém, a necessidade de uma espessura de de equilíbrio eletrônico the confere algumas limitações, já que a incidência oblíqua de um feixe de radiação sobre esta espessura faz com que este penetre a uma profundidade maior que a profundidade de dose máxima, interferindo assim na obtenção de resultados confiáveis. É possível observar que, além de apresentar as maiores diferenças entre as doses medidas e as prescritas, as medições dos campos 2 e 4 apresentam sempre valores de desvio porcentual menores ou iguais a 0 , o que parece ser coerente com os resultados esperados. Acredita-se que tal problema pode ser resolvido com a utilização de um capa de equilíbrio eletrônico que permita uma espessura constante, independentemente do 
ângulo de incidência do feixe de irradiação, com por exemplo, uma capa de formato semi-esférico.

Dessa forma, considerando-se as medidas de dose dos campos para quais a dosimetria TL com a espessura de equilíbrio eletrônico utilizada é indicada (campos 1, 3 e 5), obteve-se um desvio máximo de $-11 \%$. Levando em consideração a incerteza das medições e a incerteza na prescrição de dose (2,5\%), os resultados podem ser considerados satisfatórios. 


\section{Conclusões}

O fato de que todos os dosímetros TL utilizados eram novos proporcionaram a chance de um trabalho em que se pôde conferir aos dispositivos características de resposta muito semelhantes; para um trabalho em que se pressupõe a validação de certos procedimentos com o intuito de uma implementação viável e confiável, o uso de um grupo de dispositivos com resposta homogênea é importante por descartar as variações nas características de desempenho (comuns em dosímetros com diferentes históricos de irradiação e de tratamento térmico) como possíveis motivos para eventuais desvios na comparação entre as doses medidas e as avaliadas pelo sistema de planejamento do tratamento.

Aos desvios observados nas medições no objeto simulador antropomórfico e in vivo, com base nos resultados satisfatórios obtidos nos procedimentos de estabelecimento do sistema dosimétrico, atribuem-se fatores como a dificuldade de posicionamento do conjunto formado pelo detector TL e pela espessura de equilíbrio eletrônico sobre a superfície irregular do simulador e do paciente e os ângulos entre o eixo central do feixe incidente e a normal à superfície irradiada, o que, no caso dos campos de números 2 e 4 do tratamento de câncer de próstata, compromete seriamente os resultados obtidos.

Para a implantação dos procedimentos apresentados neste trabalho, como parte de um programa de garantia da qualidade em um Serviço de Radioterapia, sugere-se, além de um criterioso cuidado no posicionamento dos detectores, o uso de recipientes com geometrias que permitam equilíbrio eletrônico em irradiações com feixes oblíquos. 


\section{Referências Bibliográficas}

1 WILLIAMS, J. R.; THWAITES, D. I. Introduction. In: WILLIAMS, J. R.; THWAITES, D. I. (Ed.). Radiotherapy physics: in practice. 2. ed. New York, NY: Oxford, 2000. p. 1-5.

2 KLEVENHAGEN, S. C.; THWAITES, D. I.; AUKETT, R. J. Kilovoltage X-rays. In: WILLIAMS, J. R.; THWAITES, D. I. (Ed.). Radiotherapy physics: in practice. 2. ed. New York, NY: Oxford, 2000. p. 99-117.

3 FISCHER, J. J.; MOULDER, J. E. Steepness of dose-response curve in radiationtherapy - theoretical considerations and experimental results. Radiology, v. 117, n. 1, p. 179-184, 1975.

4 BARTELINK, H.; BREUR, K.; HART, G. Radiotherapy of limph-node metastases in patients with squamous-cell carcinoma of the head and neck region. International Journal of Radiation Oncology Biology Physics, v. 8, n. 6, p. 983-989, 1982.

5 SMIT, W. G. J. M.; HELLE, P. A.; VANPUTTEN, W. L. J.; WIJNMAALEN, A. J.; SELDENRATH, J. J.; VANDERWERFMESSING, B. H. P. Late radiation-damage in prostate-cancer patients treated by high-dose external radiotherapy in relation to rectal dose. International Journal of Radiation Oncology Biology Physics, v. 18, n. 1, p. 23-29, 1990.

6 EMAMI, B.; LYMAN, J.; BROWN, A.; COIA, L.; GOITEIN, M.; MUNZENRIDER, J. E.; SHANK, B.; SOLIN, L. J.; WESSON, M. Tolerance of normal tissue to therapeutic irradiation. International Journal of Radiation Oncology Biology Physics, v. 21, n. 1, p. 109-122, 1991.

7 BURMAN, C.; KUTCHER, G.; EMAMI, B.; GOITEIN, M. Fitting of normal tissue tolerance data to an analytic-function. International Journal of Radiation Oncology Biology Physics, v. 21, n. 1, p. 123-135, 1991.

8 INTERNATIONAL COMMISSION ON RADIATION UNITS AND MEASUREMENTS. ICRU Report 24. Determination of absorbed dose in a patient irradiated by beams of $X$ or gamma rays in radiotherapy procedures. Washington, DC: ICRU, 1976.

9 GOITEIN, M. Nonstandard deviations. Medical Physics, v. 10, n. 5, p. 709-711, 1983.

10 BRAHME, A. Dosimetric precision requirements in radiation-therapy. Acta Radiologica Oncology, v. 23, n. 5, p. 379-391, 1984.

11 MIJNHEER, B. J.; BATTERMANN, J. J.; WAMBERSIE, A. What degree of accuracy is required and can be achieved in photon and neutron therapy? Radiotherapy and Oncology, v. 8, n. 3, p. 237-252, 1987. 
12 DUTREIX, A. When and how can we improve precision in radiotherapy? Radiotherapy and Oncology, v. 2, n. 4, p. 275-292, 1984.

13 GOITEIN, M. Calculation of the uncertainty in the dose delivery during radiation therapy. Medical Physics, v. 12, n. 5, p. 608-612, 1985.

14 ESSERS, M.; LANSON, J. H.; MIJNHEER, B. J. In vivo dosimetry during conformal therapy of prostatic cancer. Radiotherapy and Oncology, v. 29, n. 2, p. 271-279, 1993.

15 BOELLAARD, R.; HERK, M. van; UITERWAAL, H.; MIJNHEER, B. First clinical tests using a liquid-filled electronic portal imaging device and a convolution model for the verification of the midplane dose. Radiotherapy and Oncology, v. 47, n. 3, p. 303-312, 1998.

16 KROONWIJK, M.; PASMA, K. L.; QUINT, S.; KOPER, P. C. M.; VISSER, A. G.; HEIJMEN, B. J. M. In vivo dosimetry for prostate cancer patients using an electronic portal imaging device (EPID); demonstration of internal organ motion. Radiotherapy and Oncology, v. 49, n. 2, p. 125-132, 1998.

17 LANSON, J. H.; ESSERS, M.; MEIJER, G. J.; MINKEN, A. W. H.; UITERWAAL, G. J.; MIJNHEER, B. J. In vivo dosimetry during conformal radiotherapy: requirements for and findings of a routine procedure. Radiotherapy and Oncology, v. 52, n. 1, p. 51-59, 1999.

18 BLANCO, S.; LOPEZBOTE, M. A.; DESCO, M. Quality assurance in radiation therapy: systematic evaluation of errors during the treatment execution. Radiotherapy and Oncology, v. 8, n. 3, p. 253-261, 1987.

19 LEUNENS, G.; VERSTRAETE, J.; VANDENBOGAERT, W.; VANDAM, J.; DUTREIX, A.; VANDERSCHUEREN, E. Human errors in data transfer during the preparation and delivery of radiation treatment affecting the final result - garbage in, garbage out. Radiotherapy and Oncology, v. 23, n. 4, p. 217-222, 1992.

20 NORDIC ASSOCIATION OF CLINICAL PHYSICS. Procedures in external radiation therapy dosimetry with electron and photon beams with maximum energies between 1 and $50 \mathrm{MeV}$. Recommendations by the Nordic Association of Clinical Physics (NACP). Acta Radiologica Oncology, v. 19, n. 1, p. 55-79, 1980.

21 WORLD HEALTH ORGANIZATION. Quality assurance in radiotherapy. Geneva: WHO, 1988.

22 KUTCHER, G. J.; COIA, L.; GILLIN, M.; HANSON, W. F.; LEIBEL, S.; MORTON, R. J.; PALTA, J. R.; PURDY, J. A.; REINSTEIN, L. E.; SVENSSON, G. K.; WELLER, M.; WINGFIELD, L. Comprehensive QA for radiation oncology: Report of AAPM Radiation Therapy Committee Task Group 40. Medical Physics, v. 21, n. 4, p. 581-618, 1994.

23 BRASIL. Ministério da Saúde. Agência Nacional de Vigilância Sanitária. Resolução RDC n. 20, de 2 de fevereiro de 2006. Estabelece o Regulamento Técnico para o funcionamento de serviços de radioterapia, visando a defesa da saúde dos pacientes, dos profissionais envolvidos e do público em geral. Diário Oficial da União, Poder Executivo, Brasília, 6 fev. 2006. 
24 RIZZOTTI, A.; COMPRI, C.; GARUSI, G. F. Dose evaluation to patients irradiated by Co-60 beams, by means of direct measurement on the incident and on the exit surfaces. Radiotherapy and Oncology, v. 3, n. 3, p. 279-283, 1985.

25 LEUNENS, G.; DAM, J. V.; DUTREIX, A.; SCHUEREN, E. van der. Quality assurance in radiotherapy by in vivo dosimetry. 2 . Determination of the target absorbed dose. Radiotherapy and Oncology, v. 19, n. 1, p. 73-87, 1990.

26 HEUKELOM, S.; LANSON, J. H.; MIJNHEER, B. J. In vivo dosimetry during pelvic treatment. Radiotherapy and Oncology, v. 25, n. 2, p. 111-120, 1992.

27 TERRON, J. A.; SANCHEZ-DOBLADO, F.; ARRANS, R.; SANCHEZ-NIETO, B.; ERRAZQUIN, L. Midline dose algorithm for in vivo dosimetry. Medical Dosimetry, v. 19, n. 4 , p. 263-267, 1994.

28 HUYSKENS, D.; VAN DAM, J.; DUTREIX, A. Midplane dose determination using in vivo dose measurements in combination with portal imaging. Physics in Medicine and Biology, v. 39, n. 7, p. 1089-1101, 1994.

29 BOELLAARD, R.; ESSERS, M.; HERK, M. van; MIJNHEER, B. J. New method to obtain the midplane dose using portal in vivo dosimetry. International Journal of Radiation Oncology Biology Physics, v. 41, n. 2, p. 465-474, 1998.

30 KNÖÖS, T.; AHLGREN, L.; NILSSON, M. Comparison of measured and calculated absorbed doses from tangential irradiation of the breast. Radiotherapy and Oncology, v. 7, n. 1, p. 81-88, 1986.

31 LEUNENS, G.; DAM, J. V.; DUTREIX, A.; SCHUEREN, E. van der. Quality assurance in radiotherapy by in vivo dosimetry. 1. Entrance dose measurements, a reliable procedure. Radiotherapy and Oncology, v. 17, n. 2, p. 141-151, 1990.

32 INTERNATIONAL COMMISSION ON RADIATION UNITS AND MEASUREMENTS. ICRU Report 50. Prescribing, recording and reporting photon beam therapy. Washington, DC: ICRU, 1993.

33 MARSHALL, M.; DOCHERTY, J. Measurement of skin dose from low energy beta and gamma radiation using thermoluminescent discs. Physics in Medicine and Biology, v. 16, n. 3, p. 503-510, 1971.

34 AMERICAN ASSOCIATION OF PHYSICISTS IN MEDICINE. The physical aspects of total and half body irradiation. AAPM Report No. 17. New York, N.Y.: American Institute of Physics, 1986.

35 KRON, T.; SCHNEIDER, M.; MURRAY, A.; MAMEGHAN, H. Clinical thermoluminescence dosimetry: how do expectations and results compare? Radiotherapy and Oncology, v. 26, n. 2, p. 151-161, 1993.

36 KRON, T.; ELLIOT, A.; WONG, T.; SHOWELL, G.; CLUBB, B.; METCALFE, P. X-ray surface dose measurements using TLD extrapolation. Medical Physics, v. 20, n. 3, p. 703-711, 1993.

37 RUDÉN, B. I. Evaluation of the clinical use of TLD. Acta Radiologica Therapy Physics Biology, v. 15, n. 5, p. 447-464, 1976. 
38 MCKINLAY, A. Applications of TLD in medicine. In: OBERHOFER, M.; SCHARMANN, A. (Ed.). Applied thermoluminescence dosimetry. Bristol: Adam Hilger, 1981. p. 271-287.

39 RIKNER, G.; GRUSELL, E. General specifications for silicon semiconductors for use in radiation dosimetry. Physics in Medicine and Biology, v. 32, n. 9, p. 1109-1117, 1987.

40 RIKNER, G.; GRUSELL, E. Patient dose measurements in photon fields by means of silicon semiconductor detectors. Medical Physics, v. 14, n. 5, p. 870-873, 1987.

41 HEUKELOM, S.; LANSON, J. H.; MIJNHEER, B. J. Comparison of entrance and exit dose measurements using ionization chambers and silicon diodes. Physics in Medicine and Biology, v. 36, n. 1, p. 47-59, 1991.

42 VAN DAM, J.; MARINELLO, G. Methods for in vivo dosimetry in external radiotherapy. Brussel: ESTRO, 1994.

43 METCALFE, P.; KRON, T.; HOBAN, P. Dosimetry of therapeutic X-rays. In: The physics of radiotherapy $X$-rays from linear accelerators. Madison, WI: Medical Physics Publishing, 1997. cap. 3, p. 91-228.

$44 \mathrm{KRON}, \mathrm{T}$. Applications of thermoluminescence dosimetry in medicine. Radiation Protection Dosimetry, v. 85, n. 1-4, p. 333-340, 1999.

45 MAYLES, W. P. M.; HEISIG, S.; MAYLES, H. M. O. Treatment verification and in vivo dosimetry. In: WILLIAMS, J. R.; THWAITES, D. I. (Ed.). Radiotherapy physics: in practice. 2. ed. New York, NY: Oxford, 2000. p. 220-246.

46 HUYSKENS, D.; BOGAERTS, R.; VERSTRAETE, J.; LöÖF, M.; NYSTRöM, H.; FIORINO, C.; BROGGI, S.; JORNET, N.; RIBAS, M.; THWAITES, D. Practical guidelines for the implementation of in vivo dosimetry with diodes in external radiotherapy with photon beams (entrance dose). Brussel: ESTRO, 2001.

47 THWAITES, D. I.; MIJNHEER, B. J.; MILLS, J. A. Quality assurance of external beam radiotherapy. In: PODGORSAK, E. B. (Ed.). Radiation oncology physics: a handbook for teachers and students. Vienna: IAEA, 2005. p. 407-450.

48 OLSEN, K. J.; HANSEN, J. W.; WILLE, M. Response of the alanine radiation dosemeter to high-energy photon and electron beams. Physics in Medicine and Biology, v. 35, n. 1, p. 43-52, 1990.

49 GLADSTONE, D. J.; CHIN, L. M. Automated data collection and analysis system for mosfet radiation detectors. Medical Physics, v. 18, n. 3, p. 542-548, 1991.

50 SOUBRA, M.; CYGLER, J.; MACKAY, G. Evaluation of a dual bias dual metal oxide-silicon semiconductor field effect transistor detector as radiation dosimeter. Medical Physics, v. 21, n. 4, p. 567-572, 1994.

51 GLADSTONE, D. J.; LU, X. Q.; HUMM, J. L.; BOWMAN, H. F.; CHIN, L. M. A miniature mosfet radiation dosimeter probe. Medical Physics, v. 21, n. 11, p. 1721-1728, 1994. 
52 BEDDAR, A. S.; MACKIE, T. R.; ATTIX, F. H. Water-equivalent plastic scintillation detectors for high-energy beam dosimetry: I. Physical characteristics and theoretical considerations. Physics in Medicine and Biology, v. 37, n. 10, p. 1883-1900, 1992.

53 BEDDAR, A. S.; MACKIE, T. R.; ATTIX, F. H. Water-equivalent plastic scintillation detectors for high-energy beam dosimetry: II. Properties and measurements. Physics in Medicine and Biology, v. 37, n. 10, p. 1901-1913, 1992.

54 PERERA, H.; WILLIAMSON, J. F.; MONTHOFER, S. P.; BINNS, W. R.; KLARMANN, J.; FULLER, G. L.; WONG, J. W. Rapid 2-dimensional dose measurement in brachytherapy using plastic scintillator sheet - linearity, signal-tonoise ratio, and energy response characteristics. International Journal of Radiation Oncology Biology Physics, v. 23, n. 5, p. 1059-1069, 1992.

55 VATNITSKY, S. M.; KHRUNOV, V. S.; FOMINYCH, V. I.; SCHUELE, E. Diamond detector dosimetry for medical applications. Radiation Protection Dosimetry, v. 47, n. $1-4$, p. 515-518, 1993.

56 VATNITSKY, S.; JÄRVINEN, H. Application of a natural diamond detector for the measurement of relative dose distributions in radiotherapy. Physics in Medicine and Biology, v. 38, n. 1, p. 173-184, 1993.

57 HEYDARIAN, M.; HOBAN, P. W.; BECKHAM, W. A.; BORCHARDT, I. M.; BEDDOE, A. H. Evaluation of a PTW diamond detector for electron beam measurements. Physics in Medicine and Biology, v. 38, n. 8, p. 1035-1042, 1993.

58 POLF, J. C.; MCKEEVER, S. W. S.; AKSELROD, M. S.; HOLMSTROM, S. A real-time, fibre optic dosimetry system using $\mathrm{Al}_{2} \mathrm{O}_{3}$ fibres. Radiation Protection Dosimetry, v. 100, n. 1-4, p. 301-304, 2002.

59 ANDERSEN, C. E.; AZNAR, M. C.; BØTTER-JENSEN, L.; BÄCK, Å. J.; S, S. M.; MEDIN, J. Development of optical fibre luminescence techniques for real time in vivo dosimetry in radiotherapy. In: INTERNATIONAL SIMPOSYUM ON STANDARDS AND CODES OF PRACTICE IN MEDICAL RADIATION DOSIMETRY. Standards and codes of practice in medical radiation dosimetry. Proceedings of an International Symposium held in Vienna, Austria, 25-28 November 2002. Vienna: IAEA, 2003. v. 2, p. 353-360.

60 GAZA, R.; MCKEEVER, S. W. S.; AKSELROD, M. S. Near-real-time radiotherapy dosimetry using optically stimulated luminescence of $\mathrm{Al}_{2} \mathrm{O}_{3}$ : C: mathematical models and preliminary results. Medical Physics, v. 32, n. 4, p. 1094-1101, 2005.

61 YUKIHARA, E. G.; YOSHIMURA, E. M.; LINDSTROM, T. D.; AHMAD, S.; TAYLOR, K. K.; MARDIROSSIAN, G. High-precision dosimetry for radiotherapy using the optically stimulated luminescence technique and thin $\mathrm{Al}_{2} \mathrm{O}_{3}: \mathrm{C}$ dosimeters. Physics in Medicine and Biology, v. 50, n. 23, p. 5619-5628, 2005.

62 IZEWSKA, J.; RAJAN, G. Radiation dosimeters. In: PODGORSAK, E. B. (Ed.). Radiation oncology physics: a handbook for teachers and students. Vienna: IAEA, 2005. p. 71-99. 
63 ESSERS, M.; MIJNHEER, B. J. In vivo dosimetry during external photon beam radiotherapy. International Journal of Radiation Oncology Biology Physics, v. 43, n. 2, p. 245-259, 1999.

64 CAMERON, J. R.; SUNTHARALINGAM, N.; KENNEY, G. N. Thermoluminescent dosimetry. Madison, WI: University of Wisconsin, 1968.

65 HOROWITZ, Y. S. The theoretical and microdosimetric basis of thermoluminescence and applications to dosimetry. Physics in Medicine and Biology, v. 26, n. 4, p. 765-824, 1981.

66 MCKINLAY, A. Thermoluminescence dosimetry. Bristol: Adam Hilger, 1981.

67 MCKEEVER, S. W. S.; MOSCOVITCH, M.; TOWNSEND, P. D. Thermoluminescence dosimetry materials: properties and uses. Ashford: Nuclear Technology, 1995.

68 FURETTA, C.; WENG, P.-S. Operational thermoluminescence dosimetry. Singapore: World Scientific, 1998.

69 DRISCOLL, C. M. H.; BARTHE, J. R.; OBERHOFER, M.; BUSUOLI, G.; HICKMAN, C. Annealing procedures for commonly used adiothermoluminescent materials. Radiation Protection Dosimetry, v. 14, n. 1, p. 17-32, 1986.

70 RANDALL, J. T.; WILKINS, M. H. F. Phosphorescence and electron traps. I. The study of trap distributions. Royal Society of London Proceedings. Series A: Mathematical and Physical Sciences, v. 184, n. 999, p. 365-389, 1945.

71 RANDALL, J. T.; WILKINS, M. H. F. Phosphorescence and electron traps. II. The interpretation of long-period phosphorescence. Royal Society of London Proceedings. Series A: Mathematical and Physical Sciences, v. 184, n. 999, p. 390-407, 1945.

72 DELGADO, A.; GÓMEZ-ROS, J. M. Glow curve analysis - a method for improving TLD reliability. Radiation Protection Dosimetry, v. 43, n. 1-4, p. 143-146, 1992.

73 BOS, A. J. J.; PITERS, T.; GÓMEZ-ROS, J.; DELGADO, A. An intercomparison of glow curve analysis computer programs: I. Synthetic glow curves. Radiation Protection Dosimetry, v. 47, n. 1-4, p. 473-477, 1993.

74 DELGADO, A.; GÓMEZ-ROS, J. M.; MUÑIZ, J. L. Computerised analysis of LiF GR-200 TL signals: application to dose measurements in the $\mu$ Gy range. Radiation Protection Dosimetry, v. 60, n. 2, p. 147-153, 1995.

75 KRON, T.; BUTSON, M.; HUNT, F.; DENHAM, J. TLD extrapolation for skin dose determination in vivo. Radiotherapy and Oncology, v. 41, n. 2, p. 119-123, 1996.

76 OSTWALD, P. M.; KRON, T.; HAMILTON, C. S.; DENHAM, J. W. Clinical use of carbon-loaded thermoluminescent dosimeters for skin dose determination. International Journal of Radiation Oncology Biology Physics, v. 33, n. 4, p. 943-950, 1995. 
77 SNEED, P. K.; ALBRIGHT, N. W.; WARA, W. M.; PRADOS, M. D.; WILSON, C. B. Fetal dose estimates for radiotherapy of brain tumors during pregnancy. International Journal of Radiation Oncology Biology Physics, v. 32, n. 3, p. 823-830, 1995.

78 AMIES, C. J.; MAMEGHAN, H.; ROSE, A.; FISHER, R. J. Testicular doses in definitive radiation therapy for localized prostate cancer. International Journal of Radiation Oncology Biology Physics, v. 32, n. 3, p. 839-846, 1995.

79 MARCIE, S.; COSTA, A.; LAGRANGE, J.-L. Protection of testes during radiation treatment by irregular and focused fields of $25 \mathrm{MV} \mathrm{X}$-rays: in vivo evaluation of the absorbed dose. Medical Dosimetry, v. 20, n. 4, p. 269-273, 1995.

80 KRON, T.; METCALFE, P.; WONG, T. Thermoluminescence dosimetry of therapeutic X-rays with LiF ribbons and rods. Physics in Medicine and Biology, v. 38, n. 6, p. 833-845, 1993.

81 FURETTA, C.; LEROY, C.; LAMARCHE, F. A precise investigation on the TL behavior of LiF : Mg, Cu, P (GR-200A). Medical Physics, v. 21, n. 10, p. 1605-1609, 1994.

82 ASSOCIAÇÃO BRASILEIRA DE NORMAS TÉCNICAS; INSTITUTO NACIONAL DE METROLOGIA NORMATIZAÇÃO E QUALIDADE INDUSTRIAL. Guia para a expressão da incerteza de medição. 3. ed. Rio de Janeiro: ABNT, INMETRO, 2003.

83 INTERNATIONAL ATOMIC ENERGY AGENCY. Absorbed dose determination in external beam radiotherapy: An International Code of Practice for Dosimetry Based on Standards of Absorbed Dose to Water, Technical Report Series No. 398. Vienna: IAEA, 2000. 